\title{
HOLOMORPHIC FLEXIBILITY PROPERTIES OF COMPLEX MANIFOLDS
}

\author{
FRANC FORSTNERIČ
}

\begin{abstract}
We obtain results on approximation of holomorphic maps by algebraic maps, the jet transversality theorem for holomorphic and algebraic maps between certain classes of manifolds, and the homotopy principle for holomorphic submersions of Stein manifolds to certain algebraic manifolds.
\end{abstract}

\section{INTRODUCTION}

In the present paper we use the term holomorphic flexibility property for any of several analytic properties of complex manifolds which are opposite to Kobayashi-Eisenman-Brody hyperbolicity, the latter expressing holomorphic rigidity. A connected complex manifold $Y$ is $n$-hyperbolic for some $n \in\{1, \ldots, \operatorname{dim} Y\}$ if every entire holomorphic map $\mathbb{C}^{n} \rightarrow Y$ has rank less than $n$; for $n=1$ this means that every holomorphic map $\mathbb{C} \rightarrow Y$ is constant (4], [16, 46], 47]). On the other hand, all flexibility properties of $Y$ will require the existence of many such maps.

We shall center our discussion around the following classical property which was studied by many authors (see the surveys [53] and [25]):

Oka property: Every continuous map $f_{0}: X \rightarrow Y$ from a Stein manifold $X$ is homotopic to a holomorphic map $f_{1}: X \rightarrow Y$; if $f_{0}$ is holomorphic on (a neighborhood of) a compact $\mathcal{H}(X)$-convex subset $K$ of $X$ then a homotopy $\left\{f_{t}\right\}_{t \in[0,1]}$ from $f_{0}$ to a holomorphic map $f_{1}$ can be chosen such that $f_{t}$ is holomorphic and uniformly close to $f_{0}$ on $K$ for every $t \in[0,1]$.

Here, $\mathcal{H}(X)$-convexity means convexity with respect to the algebra $\mathcal{H}(X)$ of all holomorphic functions on $X(\S 2)$. It was recently proved in [26] that the Oka property of a complex manifold $Y$ is equivalent to the following

Convex approximation property (CAP): Every holomorphic map $K \rightarrow Y$ on a compact convex set $K \subset \mathbb{C}^{n}(n \in \mathbb{N})$ can be approximated uniformly on $K$ by entire holomorphic maps $\mathbb{C}^{n} \rightarrow Y$.

In the present paper we consider relations between the Oka property and various notions of ellipticity introduced by Gromov [40] and the author [22], validity of the jet transversality theorem for holomorphic and algebraic maps (theorems 1.3 1.4

Date: May 5, 2005.

2000 Mathematics Subject Classification. Primary 32E10, 32E30, 32H02, 32J25, 32Q28; secondary 14J99, 14R10.

Key words and phrases. Stein manifolds, holomorphic maps, algebraic manifolds, algebraic maps, Oka principle, transversality, dominability.

Research supported by grants P1-0291 and J1-6173, Republic of Slovenia. 
and results in $\S 4$ ), and dominability by complex Euclidean spaces; these are summarized in corollary 1.7 Another main result concerns approximation of holomorphic maps by algebraic maps (theorem 1.1] corollary 1.2 and results in $\S 3$ ).

A principal application is the one-parametric homotopy principle for holomorphic submersions from Stein manifolds to certain quasi-projective algebraic manifolds (theorem [5.1), extending the results from 23] and 24.

An algebraic manifold (resp. an algebraic variety) will be understood in the sense of Serre ( $\S 34$ in 62, p. 226): A space $X$ endowed with a Zariski topology (each decreasing sequence of closed sets is stationary), together with a sheaf of rings $\mathcal{O}_{X}$ of continuous $\mathbb{C}$-valued functions, such that $X$ is covered by finitely many open sets $U_{j}$, each of them isomorphic (as a ringed space) to a quasi-affine algebraic manifold (resp. variety) $V_{j} \subset \mathbb{C}^{n_{j}}$ (Axiom $\mathrm{VA}_{I}$ ), with the diagonal $\Delta$ of $X \times X$ closed in $X \times X$ (Axiom $\mathrm{VA}_{I I}$ ). Here, as usual, a quasi-affine (resp. quasi-projective) variety is the difference $X=X_{0} \backslash X_{1}$ of two closed affine (resp. projective algebraic) subvarieties. Each algebraic manifold (resp. algebraic variety) $X$ has an underlying structure of a complex (holomorphic) manifold (resp. a complex space), with an induced map of $\mathcal{O}_{X}$ to the sheaf $\mathcal{H}_{X}$ of germs of holomorphic functions on $X$ 63]. All algebraic maps in this paper are assumed to be morphisms (without singularities), thus defining holomorphic maps of the underlying holomorphic manifolds.

The following notions are explained more precisely in $\S 2$ below; see especially definition 2.1. A spray on a complex manifold $Y$ is a holomorphic map $s: E \rightarrow Y$ from the total space of a holomorphic vector bundle $p: E \rightarrow Y$, satisfying $s\left(0_{y}\right)=y$ for all $y \in Y$. The spray is algebraic if $p: E \rightarrow Y$ is an algebraic vector bundle and the spray map $s: E \rightarrow Y$ is algebraic. A complex (resp. algebraic) manifold $Y$ is (algebraically) subelliptic if it admits a finite collection of (algebraic) sprays $s_{j}: E_{j} \rightarrow Y$ such that for every $y \in Y$ the vector subspaces $\left(d s_{j}\right)_{0_{y}}\left(E_{j, y}\right) \subset T_{y} Y$ together span $T_{y} Y ; Y$ is elliptic if this holds with a single (dominating) spray. Every complex homogeneous manifold is elliptic [40] (see $\S 2$ below).

We begin by discussing algebraic approximations of holomorphic maps.

Theorem 1.1. If $X$ is an affine algebraic manifold and $Y$ is an algebraically subelliptic manifold then a holomorphic map $X \rightarrow Y$ which is homotopic to an algebraic map can be approximated by algebraic maps uniformly on compacts in $X$. In particular, every null-homotopic holomorphic map $X \rightarrow Y$ is a limit of algebraic maps.

Letting $K$ be a (geometrically) convex compact set in $X=\mathbb{C}^{n}$ we obtain the following corollary.

Corollary 1.2. (Algebraic CAP) If $Y$ is an algebraically subelliptic manifold then every holomorphic map $K \rightarrow Y$ from a compact convex subset $K \subset \mathbb{C}^{n}(n \in \mathbb{N})$ can be approximated uniformly on $K$ by algebraic maps $\mathbb{C}^{n} \rightarrow Y$.

More precise results and examples can be found in $\S 2$ and $\S 3$.

The problem of approximating holomorphic maps by algebraic maps is of central importance in analytic geometry. Algebraic approximations in general do not exist even for maps between affine algebraic manifolds (for example, there are no nontrivial algebraic morphisms $\left.\mathbb{C} \rightarrow \mathbb{C}_{*}=\mathbb{C} \backslash\{0\}\right)$. Demailly, Lempert and Shiffman [14] and Lempert [54] proved that a holomorphic map from a Runge domain in an affine algebraic variety to a quasi-projective algebraic manifold can be approximated uniformly on compacts by Nash algebraic maps. (A map $U \rightarrow Y$ from an 
open set $U$ in an algebraic variety $X$ is Nash algebraic if its graph is contained in an algebraic subvariety $\Gamma \subset X \times Y$ with $\operatorname{dim} \Gamma=\operatorname{dim} X$, [57.) Nash algebraic approximations do not suffice in the proof of our theorem [5.1 where we need to approximate a holomorphic map $K \rightarrow Y$ on a compact convex set $K \subset \mathbb{C}^{n}$ by an entire map $\mathbb{C}^{n} \rightarrow Y$ whose ramification locus is a thin algebraic subvariety of $\mathbb{C}^{n}$. Global Nash algebraic maps would suffice for this purpose, but these are algebraic morphisms according to Serre (63, p. 13, proposition 8).

We shall next discuss the transversality theorems for holomorphic maps. If $X$ and $Y$ are smooth manifolds, $k \in\{0,1,2, \ldots\}$ and $Z$ is a smooth closed submanifold of $J^{k}(X, Y)$ (the manifold of $k$-jets of smooth maps of $X$ to $Y$ ) then for a generic smooth map $f: X \rightarrow Y$ its $k$-jet extension $j^{k} f: X \rightarrow J^{k}(X, Y)$ is transverse to $Z$ (Thom 68, 69; for extensions see [1, 31, 32, [55, 70, 72]). The analogous result only rarely holds for holomorphic maps. Indeed, the transversality theorem for one-jets of holomorphic maps $\mathbb{C}^{n} \rightarrow Y$ implies that all Kobayashi-Eisenman metrics on $Y$ vanish identically, and $Y$ is dominable by $\mathbb{C}^{n}, n=\operatorname{dim} Y$. If such $Y$ is compact and connected then its Kodaira dimension $\kappa=\operatorname{kod} Y$ ([2], p. 29) satisfies $\kappa<\operatorname{dim} Y$, i.e., $Y$ is not of general Kodaira type [7, 48, 49, [50.

In the positive direction, the basic transversality theorem (for 0-jets) holds for holomorphic maps to any manifold with a submersive family of holomorphic selfmaps (Abraham 1]); a classical example is Bertini's theorem to the effect that almost all projective hyperplanes in $\mathbb{P}_{n}=\mathbb{P}_{n}(\mathbb{C})$ intersect a given complex submanifold $Z \subset \mathbb{P}_{n}$ transversally (32, p. 150; 45). The jet transversality theorem holds for holomorphic maps of Stein manifolds to Euclidean spaces [19]. Kaliman and Zaidenberg [44 proved the jet transversality theorem for holomorphic maps from Stein manifolds to any complex manifold provided that one shrinks the domain of the map (theorem 4.8 below).

In $\S 4$ of this paper we prove the following transversality theorems.

Theorem 1.3. Holomorphic maps from a Stein manifold to a subelliptic manifold satisfy the jet transversality theorem with respect to closed complex analytic subvarieties. Algebraic maps from an affine algebraic manifold to an algebraically subelliptic manifold satisfy the jet transversality theorem on compact sets with respect to closed complex analytic (not necessarily algebraic) subvarieties.

Theorem 1.4. If $X$ is a Stein manifold and $Y$ is a complex manifold enjoying the Oka property then holomorphic maps $X \rightarrow Y$ satisfy the jet transversality theorem with respect to closed complex analytic subvarieties.

These follow from theorems 4.2, 4.3 and proposition 4.6 in $\S 4$ where the reader can find more precise formulations and further results. Although the part of theorem 1.3 for holomorphic maps is implied by theorem 1.4 and the fact that subellipticity implies the Oka property [22, our proof of theorem 1.3 is more elementary than the proof of the latter implication in 22 and it also applies in the algebraic category where the Oka property is unavailable; for these reasons we separate them.

A property of a holomorphic map $X \rightarrow Y$ is said to be generic if it holds for all maps in a certain set of the second category in the Frèchét space $\mathcal{H}(X, Y)$ of all holomorphic maps $X \rightarrow Y$. The jet transversality theorem implies that singularities of a generic map $f \in \mathcal{H}(X, Y)$ (points of nonmaximal rank) satisfy the codimension conditions in [19], proposition 2. This implies the following; for the last statement 
concerning injective immersions one needs a multi-jet transversality theorem which is an easy extension (see 19, $\S 1.3$ for the case $Y=\mathbb{C}^{N}$ ).

Corollary 1.5. If $X$ is a Stein manifold and $Y$ enjoys the Oka property then a generic holomorphic map $X \rightarrow Y$ is an immersion when $\operatorname{dim} Y \geq 2 \operatorname{dim} X$ and an injective immersion when $\operatorname{dim} Y \geq 2 \operatorname{dim} X+1$.

A holomorphic map $\pi: Y \rightarrow Y_{0}$ is called a subelliptic Serre fibration if it is a surjective subelliptic submersion (definition 2.1 below) and a Serre fibration, i.e., it enjoys the homotopy lifting property (71, p. 8). Examples include holomorphic fiber bundles with subelliptic fibers and, more generally, subelliptic submersions which are topological fiber bundles (such as the unramified elliptic fibrations without exceptional fibers; see [2], p. 200). By theorem 1.8 in [26], CAP ascends and descends in a subelliptic Serre fibration, in the sense that the manifolds $Y$ and $Y_{0}$ satisfy CAP at the same time. (See also [40, 3.3.C' and 3.5.B", and [51, [52.) For holomorphic fiber bundles the same conclusion holds if the fiber satisfies CAP. A finite induction and the equivalence $\mathrm{CAP} \Leftrightarrow$ Oka property imply the following result.

Corollary 1.6. Let $Y=Y_{m} \rightarrow Y_{m-1} \rightarrow \cdots \rightarrow Y_{0}$ where every map $Y_{j} \rightarrow Y_{j-1}$ $(j=1,2, \ldots, m)$ is a subelliptic Serre fibration. If one of the manifolds $Y_{j}$ enjoys the Oka property then all of them do. This holds in particular if $\operatorname{dim} Y_{0}=0$, and in such case $Y$ will be called semisubelliptic.

If $\pi: Y \rightarrow Y_{0}$ is a ramified holomorphic map then the Oka property of $Y_{0}$ need not imply the Oka property of $Y$. (A meromorphic function on a compact hyperbolic Riemann surface $Y$ is a finite ramified holomorphic map $Y \rightarrow Y_{0}=\mathbb{P}_{1}$, the Oka property holds for $\mathbb{P}_{1}$ but fails for $Y$. See also example 6.3 in $\S 6$.) We don't know whether the Oka property of $Y$ implies the same for $Y_{0}$ (problem 6.7).

Recall that a $p$-dimensional complex manifold $Y$ is (holomorphically) dominable by $\mathbb{C}^{p}$ if there exists a holomorphic map $f: \mathbb{C}^{p} \rightarrow Y$ with rank $p$ at $0 \in \mathbb{C}^{p}$; if $Y$ and $f$ are algebraic then $Y$ is algebraically dominable by $\mathbb{C}^{p}$. If $Y$ is compact and connected then dominability by $\mathbb{C}^{p}$ implies $\operatorname{kod} Y<p=\operatorname{dim} Y$ [50].

Corollary 1.7. (Hierarchy of holomorphic flexibility properties) The following implications hold for every complex manifold:

homogeneous $\Longrightarrow$ elliptic $\Longrightarrow$ subelliptic $\Longrightarrow$ semisubelliptic $\Longrightarrow$

$\mathrm{CAP} \Longleftrightarrow$ Oka property $\Longrightarrow$ jet transversality theorem $\Longrightarrow$ dominable.

In the algebraic category we have the implications

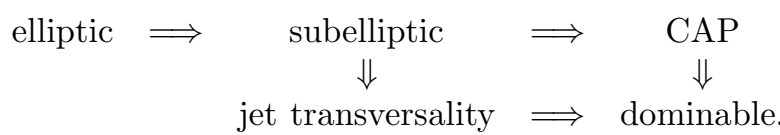

The 'jet transversality theorem' refers to holomorphic maps from any Stein manifold to $Y$. In the algebraic category CAP is interpreted in the sense of corollary 1.2 and the first vertical arrow in the last display is theorem 1.3

Clearly CAP of $Y$ implies the following strong dominability: For every point $y \in Y$ there is a holomorphic map $f_{y}: \mathbb{C}^{p} \rightarrow Y(p=\operatorname{dim} Y)$ such that $f_{y}(0)=y$ and $d f_{y}$ has maximal rank $p$ at $0 \in \mathbb{C}^{p}$. (Note that a family $\left\{f_{y}\right\}_{y \in Y}$ of such maps which depend holomorphically on the base point $y \in Y$ is a dominating spray on $Y$.) 
If $Y$ is strongly dominable by $\mathbb{C}^{p}(p=\operatorname{dim} Y)$ then every bounded plurisubharmonic function on $Y$ is constant. If such $Y$ is also Stein then every $\mathbb{R}$-complete holomorphic vector field on $Y$ is also $\mathbb{C}$-complete, i.e., it induces a holomorphic action of $(\mathbb{C},+)$ (corollary 2.2 in [21]). This implies the following.

Corollary 1.8. If a Stein manifold $Y$ enjoys CAP then every $\mathbb{R}$-complete holomorphic vector field on $Y$ is also $\mathbb{C}$-complete.

Remark 1.9. (CAP and Oka-type properties) A more precise term for the Oka property as defined above is the basic Oka property with approximation, where 'basic' refers to the non-parametric case. More general Oka-type properties have been studied by many authors (see e.g. [33, 34, 35], 8, 36, [20, [53, 40, 44, [73, [43, 28, 229, 30, 22], [51, 52]). The basic Oka property with (jet) interpolation of $Y$ refers to the possibility of homotopically deforming a continuous map $f_{0}: X \rightarrow Y$ from any Stein manifold $X$ to a holomorphic map $f_{1}: X \rightarrow Y$, keeping it fixed (to any finite order in the jet case) on a closed complex subvariety $X_{0} \subset X$ along which $f_{0}$ is holomorphic. Combining interpolation with approximation on compact $\mathcal{H}(X)$-convex subsets one obtains the Oka property with (jet) interpolation and approximation. By corollary 1.4 in [27] all these properties are equivalent to $\mathrm{CAP}$, and hence the conclusion of corollary [1.6 above holds for all mentioned Oka-type properties. The analogous equivalences hold for the parametric Oka properties (theorem 5.1 in [26] and theorem 6.1 in [27]). All these Oka properties (also the parametric versions) are implied by ellipticity [40] and subellipticity [22], and they are equivalent to ellipticity on a Stein manifold (40, 3.2.A.; proposition 1.2 in [30]).

Remark 1.10. As pointed out in [26], CAP is a localization (=restriction) of the Oka property to model pairs $-K$ a compact convex set in $X=\mathbb{C}^{n}$. The equivalence $\mathrm{CAP} \Leftrightarrow$ Oka property supports the following heuristic principle; compare with the formulation of the Oka principle by Grauert and Remmert ([37, p. 145).

Localization principle: Problems concerning holomorphic maps from Stein manifolds have only homotopical obstructions provided that the target manifold satisfies a suitable flexibility property expressed in terms of holomorphic maps from Euclidean spaces.

This principle, once established for a certain class of maps, can substantially simplify the analysis in concrete examples as is amply demonstrated in 26] and in $\S 6$ below. On the philosophical level it reveals the interesting but not entirely surprisig fact that many complex analytic problems involving Stein manifolds reduce to problems on Euclidean spaces (which does not necessarily make them easy).

By [26] the localization principle holds for sections of holomorphic fiber bundles over any Stein manifold, the corresponding local property being CAP of the fiber. Many classical problems on Stein manifolds, including the classification of principal holomorphic fiber bundles and their associated bundles with homogeneous fibers, fits into this framework (see Grauert [35], Cartan [8], and the survey [53] of Leiterer).

One may also consider special classes of holomorphic maps such as immersions, submersions, maps of constant rank, etc. According to 24] the localization principle holds for holomorphic submersions $X \rightarrow Y$ from Stein manifolds $X$, the corresponding local property of $Y$ being Property $\mathrm{S}_{\mathrm{n}}$ with $n=\operatorname{dim} X \geq \operatorname{dim} Y$ (see definition 
5.3 and theorem 5.1] in $\S 5$ below). For holomorphic immersions a localization principle is not known at this time, but the homotopy principle for immersions of Stein manifolds to Euclidean spaces was proved by Eliashberg and Gromov [17, 39].

The flexibility properties discussed in this paper are mutually exclusive with any kind of hyperbolicity property (for the latter see e.g. 4, 16, 46, 47, 74 and the surveys [10, 11, 58, 65. In particular, none of the flexibility properties holds for compact complex manifolds of Kodaira general type; it is conjectured that every such manifold $Y$ is almost hyperbolic in the sense that there is a proper complex subvariety $Y_{0} \subset Y$ containing the image of any nonconstant holomorphic map $\mathbb{C} \rightarrow Y$ (the Green-Griffiths conjecture, 38]). In $\S 6$ we consider flexibility properties of complex curves and surfaces; in the latter case our analysis rests upon the fairly complete results of Buzzard and Lu [6] on dominability of compact complex surfaces with less than maximal Kodaira dimension.

\section{Preliminaries}

We denote by $\mathcal{H}_{X}$ the sheaf of germs of holomorphic functions on a complex (holomorphic) manifold $X$, and by $\mathcal{H}(X)=\mathcal{H}(X, \mathbb{C})$ the algebra of all global holomorphic functions on $X$. Given a pair of complex manifolds $X, Y$, we denote by $\mathcal{H}(X, Y)$ the set of all holomorphic maps $X \rightarrow Y$, endowed with the compact-open topology. This topology is induced by a complete metric and hence $\mathcal{H}(X, Y)$ is a Baire space. A property of $f \in \mathcal{H}(X, Y)$ is said to be generic if it holds for all $f$ in a residual subset (a countable intersection of open dense subsets) of $\mathcal{H}(X, Y)$.

A function (or a map) is said to be holomorphic on a compact set $K$ in a complex manifold $X$ if it is holomorphic in an open set $U \subset X$ containing $K$. A homotopy of maps $\left\{f_{t}\right\}$ is holomorphic on $K$ if there is an open neighborhood $U$ of $K$, independent of $t$, such that $f_{t}$ is holomorphic on $U$ for every $t$.

An affine manifold is a closed complex or algebraic submanifold of a complex Euclidean space; an affine complex manifold is the same thing as a Stein manifold according to the embedding theorems [3], 18, [56], [59, 61].

A compact subset $K$ in a Stein manifold $X$ is $\mathcal{H}(X)$-convex (holomorphically convex in $X)$ if for any $p \in X \backslash K$ there exists $f \in \mathcal{H}(X)$ with $|f(p)|>\sup _{K}|f|$. If $X$ is embedded in $\mathbb{C}^{N}$ then a set $K \subset X$ is $\mathcal{H}(X)$-convex if and only if it is $\mathcal{H}\left(\mathbb{C}^{N}\right)$-convex, i.e., polynomially convex.

We denote by $\mathcal{O}_{X}$ the structure sheaf of an algebraic manifold $X$ and by $\mathcal{O}(X)$ the algebra of all regular algebraic functions on $X$. As we have already said in $\S 1$, an algebraic manifold or variety will be understood in the sense of Serre ( $\$ 34$ in 62. p. 226); in particular, there is an underlying complex manifold (resp. analytic variety) structure on $X$. By $\mathcal{O}(X, Y)$ we denote the set of all regular algebraic maps (morphisms) between a pair of algebraic manifolds. Clearly $\mathcal{O}(X, Y) \subset \mathcal{H}(X, Y)$ but $\mathcal{O}(X, Y)$ need not be closed in $\mathcal{H}(X, Y)$. If both $X$ and $Y$ are projective algebraic then $\mathcal{O}(X, Y)=\mathcal{H}(X, Y)$ by Sere's GAGA principle [63.

A fiber-spray associated to a holomorphic submersion $h: Y \rightarrow Y^{\prime}$ is a triple $(E, p, s)$ consisting of a holomorphic vector bundle $p: E \rightarrow Y$ and a holomorphic map $s: E \rightarrow Y$ such that for each $y \in Y$ we have $s\left(0_{y}\right)=y$ and $s\left(E_{y}\right) \subset Y_{h(y)}=$ $h^{-1}(h(y))$ (see [40], §1.1.B., and [28]). A spray on a complex manifold $Y$ is a 
fiber-spray associated to the trivial submersion $Y \rightarrow$ point. For each $y \in Y$ let $V T_{y} Y=\operatorname{ker} d h_{z} \subset T_{y} Y$, the vertical tangent space of $Y$ with respect to $h$.

Definition 2.1. A holomorphic submersion $h: Y \rightarrow Y^{\prime}$ is subelliptic if each point in $Y^{\prime}$ has an open neighborhood $U \subset Y^{\prime}$ such that the restricted submersion $h:\left.Y\right|_{U}=$ $h^{-1}(U) \rightarrow U$ admits finitely many fiber-sprays $\left(E_{j}, p_{j}, s_{j}\right)(j=1, \ldots, k)$ satisfying

$$
\left(d s_{1}\right)_{0_{y}}\left(E_{1, y}\right)+\left(d s_{2}\right)_{0_{y}}\left(E_{2, y}\right) \cdots+\left(d s_{k}\right)_{0_{y}}\left(E_{k, y}\right)=V T_{y} Y
$$

for each $\left.y \in Y\right|_{U}$; such a collection of sprays is said to be dominating. The submersion is elliptic if the above holds with $k=1$. A complex manifold $Y$ is (sub-)elliptic if the trivial submersion $Y \rightarrow$ point is such. An algebraic manifold $Y$ is algebraically subelliptic if it admits finitely many algebraic sprays satisfying the domination condition (2.1) for the trivial submersion $Y \rightarrow$ point; $Y$ is algebraically elliptic if it admits a dominating algebraic spray.

Examples of (sub-)elliptic manifolds and submersions can be found in 35] (especially sections 0.5 . B and 3.4.F), 22, 28 . If a complex Lie group $G$, with Lie algebra $\mathbf{g}$, acts holomorphically and transitively on a complex manifold $Y$ then the map $Y \times \mathbf{g} \rightarrow Y,(y, v) \rightarrow e^{v} y$, is a dominating spray on $Y$. Furthermore, every algebraic Lie group $G$ which admits no homomorphisms to $\mathbb{C}_{*}$ is algebraically elliptic; the following proof has been explained to me by J. Winkelmann. The condition on $G$ implies that it is generated by its unipotent subgroups. Each left invariant vector field on $G$ arising from a unipotent subgroup gives rise to an algebraic spray on $G$. Under the above generation condition these vector fields span the tangent space of $G$ at each point, and the composition of their flows gives a dominating algebraic spray on $G$. The Lie group $\mathbb{C}_{*}$ is not algebraically elliptic.

The following result from 22 (which is implicitly contained in lemmas 3.5.B. and 3.5.C. in [40]) will be important for us.

Proposition 2.2. Let $Y$ be a quasi-projective algebraic manifold. If every point of $Y$ has a Zariski open neighborhood $U \subset Y$ such that $U$ is algebraically subelliptic then $Y$ is itself algebraically subelliptic.

The following classes of algebraic manifolds will be used in $\S 5$.

Definition 2.3. Let $Y$ be a quasi-projective algebraic manifold.

(a) $Y$ is of Class $\mathcal{A}_{0}$ if it is covered by finitely many Zariski open sets biregularly isomorphic to the affine space $\mathbb{C}^{p}$ with $p=\operatorname{dim} Y$.

(b) $Y$ is of Class $\mathcal{A}$ if $Y=\widehat{Y} \backslash A$ where $\widehat{Y}$ is of class $\mathcal{A}_{0}$ and $A$ is a thin (=of codimension at least two) algebraic subvariety of $\widehat{Y}$.

Thus a $p$-dimensional manifold of Class $\mathcal{A}$ is Zariski locally equivalent to $\mathbb{C}^{p} \backslash A$ where $A$ is a thin algebraic subvariety in $\mathbb{C}^{p}$, depending on the chosen Zariski open set in $Y$. Such manifold $\mathbb{C}^{p} \backslash A$ is algebraically elliptic (28], p. 119; 40]). Indeed, there is a polynomial spray $s: \mathbb{C}^{p} \times \mathbb{C}^{N} \rightarrow \mathbb{C}^{p}$ which maps $\left(\mathbb{C}^{p} \backslash A\right) \times \mathbb{C}^{N}$ to $\mathbb{C}^{p} \backslash A$ and is dominating at every point $\mathbb{C}^{p} \backslash A$; such $s$ is obtained by composing flows of suitably chosen algebraic shear vector fields on $\mathbb{C}^{p}$ which vanish on $A$ and generate the tangent bundle at each point of $\mathbb{C}^{p} \backslash A$. Hence proposition 2.2 implies

Corollary 2.4. Every manifold of Class $\mathcal{A}$ is algebraically subelliptic. 
Class $\mathcal{A}_{0}$ includes all complex affine and projective spaces, as well as Grassmanians. Another example is the total space $W$ of a holomorphic fiber bundle $\pi: W \rightarrow Y$ where the base $Y$ is a manifold of Class $\mathcal{A}_{0}$, the fiber is $F=\pi^{-1}(y) \in\left\{\mathbb{C}^{m}, \mathbb{P}_{m}\right\}$, and the structure group is $G L_{m}(\mathbb{C})$ respectively $P G L_{m}(\mathbb{C})$. Every such bundle is algebraic by GAGA 63 , and its restriction to any affine Zariski open set $\mathbb{C}^{p} \simeq U \subset Y$ is algebraically trivial, $\pi^{-1}(U) \simeq U \times F$. Hence $W$ is covered by Zariski open sets biregularly isomorphic to $\mathbb{C}^{p+m}$, i.e., $W$ is of Class $\mathcal{A}_{0}$. An example are the Hirzebruch surfaces $H_{l}, l=0,1,2, \ldots\left(\left[2\right.\right.$, p. 191); these are $\mathbb{P}_{1}$-bundles over $\mathbb{P}_{1}$, and each of them is birationally equivalent to $\mathbb{P}_{2}$.

Manifolds of Class $\mathcal{A}$ have been considered by Gromov who called them Ellregular and showed that this class is stable under blowing up points ([40, §3.5.D"):

Proposition 2.5. (40]) If $Y$ is of Class $\mathcal{A}$ (respectively of Class $\mathcal{A}_{0}$ ) then any manifold obtained from $Y$ by blowing up finitely many points is also of Class $\mathcal{A}$ (respectively of Class $\mathcal{A}_{0}$ ).

Proof. By localization it suffices to show that the manifold $L$, obtained by blowing up $\mathbb{C}^{q}$ at the origin, is of Class $\mathcal{A}$. $L$ is the total space of a holomorphic line bundle $\pi: L \rightarrow \mathbb{P}_{q-1}$ (the universal bundle); $L$ is trivial over the complement of each hyperplane $\mathbb{P}_{q-2} \subset \mathbb{P}_{q-1}$ (which equals $\mathbb{C}^{q-1}$ ), and hence every point in $L$ has a Zariski neighborhood of the form $\pi^{-1}\left(\mathbb{P}_{q-1} \backslash \mathbb{P}_{q-2}\right)$ which is biregular to $\mathbb{C}^{q}$.

\section{Algebraic approximation}

All algebraic maps are assumed to be morphisms (without singularities).

Theorem 3.1. Let $X$ be an affine algebraic manifold and $K$ a compact $\mathcal{H}(X)$ convex subset of $X$. Let $Y$ be an algebraically subelliptic manifold (def. 2.1) with a distance function $d$ induced by a Riemannian metric. If $f_{t}: K \rightarrow Y(t \in[0,1])$ is a homotopy of holomorphic maps such that $f_{0}$ extends to an algebraic map $X \rightarrow Y$ then for every $\epsilon>0$ there exists an algebraic map $F: X \times \mathbb{C} \rightarrow Y$ such that $F(\cdot, 0)=f_{0}$ and $d\left(F(x, t), f_{t}(x)\right)<\epsilon$ for every $x \in K$ and $t \in[0,1]$.

Corollary 3.2. If $K \subset X$ and $Y$ are as in theorem 3.1 then every null-homotopic holomorphic map $K \rightarrow Y$ can be approximated uniformly on $K$ by algebraic maps $X \rightarrow Y$. In particular, if $K$ is a compact convex set in $\mathbb{C}^{n}$ then every holomorphic map $K \rightarrow Y$ can be approximated uniformly on $K$ by algebraic maps $\mathbb{C}^{n} \rightarrow Y$.

Examples of algebraically subelliptic manifolds are given by propositions $2.2,2.5$ and corollary 2.4. We do not know whether every homotopy class of maps $X \rightarrow Y$ in theorem 3.1 contains an algebraic map.

Theorem 3.1 is a special case (with $Z=X \times Y \rightarrow X$ ) of the following.

Theorem 3.3. Let $h: Z \rightarrow X$ be an algebraic submersion from an algebraic manifold $Z$ onto an affine algebraic manifold $X$. Let $d$ be a distance function on $Z$ induced by a Riemannian metric. Assume that $Z$ admits a family of algebraic fiber-sprays $\left(E_{j}, p_{j}, s_{j}\right)(j=1, \ldots, k)$ satisfying the domination property (2.1) at every point $z \in Z$. Let $K \subset X$ be a compact $\mathcal{H}(X)$-convex set and let $f_{t}: K \rightarrow Z$ $(t \in[0,1])$ be a homotopy of holomorphic sections such that $f_{0}$ extends to an algebraic section $X \rightarrow Z$. For every $\epsilon>0$ there is an algebraic map $F: X \times \mathbb{C} \rightarrow Z$ such that $h(F(x, t))=x$ for $(x, t) \in X \times \mathbb{C}, F(\cdot, 0)=f_{0}$, and $d\left(F(x, t), f_{t}(x)\right)<\epsilon$ for every $(x, t) \in K \times[0,1]$. 
An algebraic submersion satisfying the hypotheses of theorem 3.3 will be called algebraically subelliptic (compare with def. 2.11). Theorem 3.3 is an algebraic analogue of theorem 3.1 in [22]; see also [40] and theorems 4.1 and 4.2 in [28].

Proof of theorem 3.3 Let $(E, p, s)$ be the composed (algebraic) fiber-spray on $Z$ obtained from the fiber-sprays $\left(E_{j}, p_{j}, s_{j}\right)(j=1, \ldots, k)$ (see [40, $\S 1.3$, and [28], definition 3.3). We briefly recall the construction. Set $\left(E^{(1)}, p^{(1)}, s^{(1)}\right)=$ $\left(E_{1}, p_{1}, s_{1}\right)$. If $k>1$, let $E^{(2)}=s^{(1) *}\left(E_{2}\right) \rightarrow E^{(1)}$ (the pull-back of $p_{2}: E_{2} \rightarrow Z$ by the spray map $s^{(1)}: E^{(1)} \rightarrow Z$ ), and define $p^{(2)}: E^{(2)} \rightarrow Z$ and $s^{(2)}: E^{(2)} \rightarrow Z$ by $p^{(2)}\left(e_{1}, e_{2}\right)=p_{1}\left(e_{1}\right), s^{(2)}\left(e_{1}, e_{2}\right)=s_{2}\left(e_{2}\right)$. (Note that $s_{1}\left(e_{1}\right)=p_{2}\left(e_{2}\right)$.) Continuing inductively we obtain a sequence of algebraic vector bundle projections

$$
E=E^{(k)} \longrightarrow \cdots \longrightarrow E^{(2)} \longrightarrow E^{(1)} \longrightarrow Z .
$$

The composed bundle $E \rightarrow Z$ with fibers $E_{z}(z \in Z)$ has a well defined zero section which we identify with $Z$, and $\left.T E\right|_{Z} \simeq T Z \oplus E_{1} \oplus \cdots \oplus E_{k}$. Denote by $s=$ $s^{(k)}: E \rightarrow Z$ the composed spray. The restriction of its differential $d s_{0_{z}}: T_{0_{z}} E \rightarrow$ $T_{z} Z$ to the fiber $E_{j, z}$ of $E_{j}$ over $z$ in the above direct sum decomposition equals $\left(d s_{j}\right)_{0_{z}}: T_{0_{z}} E_{j, z} \rightarrow V T_{z} Z$. Hence (2.1) is equivalent to the domination property of the composed spray:

$$
(d s)_{0_{z}}\left(T_{0_{z}} E_{z}\right)=V T_{z} Z, \quad z \in Z .
$$

The bundle $E \rightarrow Z$ admits a (noncanonical) holomorphic vector bundle structure over any Stein subset of $Z(40], \S 1.3$, and [28], corollary 3.5).

In the remainder of the proof we shall only work with the composed spray bundle $(E, p, s)$ and will no longer need the individual sprays $\left(E_{j}, p_{j}, s_{j}\right)$.

Lemma 3.4. Let $V \subset \subset U$ be open Stein neighborhoods of $K$ in $X$ such that the homotopy $f_{t}(t \in[0,1])$ in theorem 3.3 is defined in $U$. Set $V_{t}=f_{t}(V) \subset Z$ for $t \in[0,1]$. There are numbers $l \in \mathbb{N}$ and $0=t_{0}<t_{1}<\cdots<t_{l}=1$ such that for every $j=0,1, \ldots, l-1$ there exists a homotopy of holomorphic sections $\xi_{t}$ of the restricted bundle $\left.E\right|_{V_{t_{j}}} \rightarrow V_{t_{j}}\left(t \in I_{j}=\left[t_{j}, t_{j+1}\right]\right)$ such that $\xi_{t_{j}}$ is the zero section and $s\left(\xi_{t}(z)\right)=f_{t}(h(z))$ for all $t \in I_{j}$ and $z \in V_{t_{j}}$.

Proof. Assume first that there exists a Stein open set $\Omega \subset Z$ containing $\cup_{t \in[0,1]} \bar{V}_{t}$. By corollary 3.5 in 28] the restriction $\left.E\right|_{\Omega} \rightarrow \Omega$ admits the structure of a holomorphic vector bundle and a holomorphic direct sum splitting $\left.E\right|_{\Omega}=H \oplus H^{\prime}$, where $H^{\prime}$ is the kernel of $d s$ at the zero section of $E$ (41], p. 256, theorem 7). It follows from (3.1) that for every $z \in \Omega$ the restriction $s: H_{z} \rightarrow Z_{h(z)}=h^{-1}(h(z))$ maps a neighborhood of $0_{z}$ in $H_{z}$ biholomorphically onto a relative neighborhood of $z$ in the fiber $Z_{h(z)}$. The size of this neighborhood, and of its image in the corresponding fiber of $Z$, can be chosen uniform for points in the compact set $\cup_{t \in[0,1]} \bar{V}_{t} \subset \Omega$. Hence there is a $\delta>0$ such that for every $t \in[0,1]$ the local inverse of $s:\left.H\right|_{V_{t}} \rightarrow Z$ at the zero section gives a homotopy of sections $\xi_{\tau}$ of $\left.H\right|_{V_{t}}\left(\tau \in J_{t}=[t, t+\delta] \cap[0,1]\right)$, with $\xi_{t}$ the zero section, such that $s\left(\xi_{\tau}(z)\right)=f_{\tau}(h(z))$ for $\tau \in J_{t}$ and $z \in V_{t}$. This proves lemma 3.4 in the special case.

For the general case observe that $f_{t}(U)$, being a closed Stein submanifold of $\left.Z\right|_{U}$, admits an open Stein neighborhood in $Z$ 64, 9]. By compactness there are Stein open sets $\Omega_{j} \subset Z(j=1,2, \ldots, m)$ and a partitition $[0,1]=\cup_{j=1}^{m} I_{j}$ into adjacent closed subintervals $I_{j}$ such that $\cup_{t \in I_{j}} \bar{V}_{t} \subset \Omega_{j}$. It remains to apply the above argument separately for each $I_{j}$. 
Denote by $\left(E^{(l)}, p^{(l)}, s^{(l)}\right)$ the $l$-th iterated bundle of $(E, p, s)$ (definition 3.3 in 28, p. 132; this is just the $l$-tuple composition of $(E, p, s)$ with itself). Let $\left(E^{\prime}, p^{\prime}, s^{\prime}\right)$ denote the pull-back of $\left(E^{(l)}, p^{(l)}, s^{(l)}\right)$ to $X$ by the algebraic map $f_{0}: X \rightarrow$ $Z$; by the construction this is an algebraic composed spray bundle over $X$.

Lemma 3.5. There is a homotopy $\eta_{t}:\left.V \rightarrow E^{\prime}\right|_{V}(t \in[0,1])$ of holomorphic sections of the restricted bundle $\left.E^{\prime}\right|_{V} \rightarrow V$ such that $\eta_{0}$ is the zero section and $s^{\prime}\left(\eta_{t}(x)\right)=f_{t}(x)$ for every $x \in V$ and $t \in[0,1]$.

Proof. It suffices to assemble the individual homotopies $\left\{\xi_{t}: t \in\left[t_{j}, t_{j+1}\right]\right\}(j=$ $0, \ldots, l-1$ ), furnished by lemma 3.4 into a homotopy of sections $\widetilde{\xi}_{t}:\left.V_{0} \rightarrow E^{(l)}\right|_{V_{0}}$ $(t \in[0,1])$ of the iterated bundle $E^{(l)}$ over the open subset $V_{0}=f_{0}(V)$ in the algebraic submanifold $f_{0}(X)$ of $Z$. Clearly $\widetilde{\xi}_{t}$ corresponds to a holomorphic sections $\eta_{t}: V \rightarrow E^{\prime}=f_{0}^{*}\left(E^{(l)}\right)$ of the pull-back bundle, with $\eta_{0}$ being the zero section. (For further details see [28, proposition 3.6 on p. 134.)

Lemma 3.6. Let $d^{\prime}$ be a distance function on $E^{\prime}$ induced by a Riemannian metric. Let $\left\{\eta_{t}\right\}_{t \in[0,1]}$ be as in lemma 3.5. For every $\delta>0$ there is an algebraic map $\eta^{\prime}: X \times \mathbb{C} \rightarrow E^{\prime}$ satisfying

(i) $\eta^{\prime}(x, 0)=0_{x} \in E_{x}^{\prime}(x \in X)$, and

(ii) $d^{\prime}\left(\eta^{\prime}(x, t), \eta_{t}(x)\right)<\delta$ for all $x \in K$ and $t \in[0,1]$.

Proof. By construction of the composed bundle $E^{\prime} \rightarrow X$ there is a finite sequence

$$
E^{\prime}=E^{m, 0} \longrightarrow E^{m-1,0} \longrightarrow \cdots \longrightarrow E^{1,0} \longrightarrow X
$$

with $m=k l$ and $E^{(1,0)}=f_{0}^{*} E_{1} \rightarrow X$, in which every map $E^{j, 0} \rightarrow E^{j-1,0}$ is an algebraic vector bundle projection. Here $k$ is the number of the initial sprays in theorem 3.3 and $l$ is the number in lemma 3.4

Since $X$ is an affine algebraic manifold, the algebraic vector bundle $E^{1,0} \rightarrow X$ is generated by finitely many (say $n_{1}$ ) algebraic sections according to Serre's theorem A $\left(62\right.$, p. 237, Théorème 2). This gives a surjective algebraic map $\pi_{1}: E^{1,1}=$ $X \times \mathbb{C}^{n_{1}} \rightarrow E^{1,0}$ of the trivial rank $n_{1}$ bundle onto $E^{1,0}$. Pulling back the sequence (3.2) to the new total space $E^{1,1}$ we obtain a commutative diagram

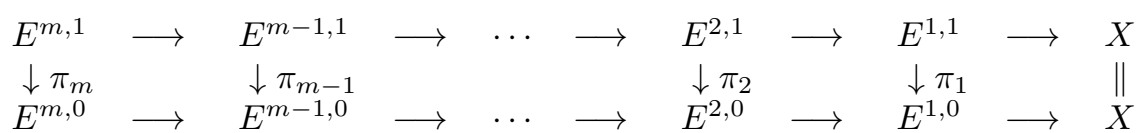

in which all horizontal maps are algebraic vector bundle projections and the vertical maps $\pi_{j}$ for $j \geq 2$ are the induced natural maps which are bijective on fibers. More precisely, we begin by letting $E^{2,1} \rightarrow E^{1,1}$ be the pull-back of the vector bundle $E^{2,0} \rightarrow E^{1,0}$ (in the bottom row) by the vertical morphism $\pi_{1}: E^{1,1} \rightarrow E^{1,0}$, and we denote by $\pi_{2}: E^{2,1} \rightarrow E^{2,0}$ the asociated natural map which makes the respective diagram commute. Moving one step to the left, $E^{3,1} \rightarrow E^{2,1}$ is the pull-back of the bundle $E^{3,0} \rightarrow E^{2,0}$ in the bottom row by the vertical morphism $\pi_{2}: E^{2,1} \rightarrow E^{2,0}$, and $\pi_{3}: E^{3,1} \rightarrow E^{3,0}$ is the associated natural map; etc. There is an algebraic spray map $s^{1}: E^{m, 1} \rightarrow Z$ which is the composition of $\pi_{m}: E^{m, 1} \rightarrow E^{m, 0}$ with the initial spray $s: E^{m, 0}=E \rightarrow Z$.

We claim that the homotopy $\eta_{t}$ of holomorphic sections of $\left.E^{m, 0}\right|_{V}=\left.E^{\prime}\right|_{V} \rightarrow V$, furnished by lemma 3.5 lifts to a homotopy $\eta_{t}^{1}$ of holomorphic sections of $\left.E^{m, 1}\right|_{V} \rightarrow$ $V$ such that $s^{1}\left(\eta_{t}^{1}\right)=f_{t}$ for all $t \in[0,1]$, and $\eta_{0}^{1}$ is the zero section. It suffices to 
see that the $E^{1,0}$-component of $\eta_{t}$ (i.e., the projection of $\eta_{t}$ under the composed projection $E^{m, 0} \rightarrow E^{1,0}$ ) lifts to $E^{1,1}$; the rest of the lifting is then obtained by applying the inverses of the fiberwise isomorphic vertical maps. But this follows from the fact that the surjective vector bundle map $\pi_{1}: E^{1,1} \rightarrow E^{1,0}$ admits a holomorphic splitting $\sigma_{1}: E^{1,0} \rightarrow E^{1,1}$ over $X$, with $\pi_{1} \circ \sigma_{1}$ the identity on $E^{1,0}$ (theorem 7 in 41, p. 256).

Repeating the same argument with the bundle $E^{2,1} \rightarrow E^{1,1}=X_{1}$ over the affine manifold $X_{1}=X \times \mathbb{C}^{n_{1}}$ we obtain a surjective algebraic vector bundle map $E^{2,2}=X_{1} \times \mathbb{C}^{n_{2}}=X \times \mathbb{C}^{n_{1}+n_{2}} \rightarrow E^{2,1}$. As before we lift the top line in the above diagram to a new level

$$
E^{m, 2} \longrightarrow E^{m-1,2} \longrightarrow \cdots \longrightarrow E^{2,2} \longrightarrow E^{1,1}=X_{1}=X \times \mathbb{C}^{n_{1}} .
$$

Note that $E^{2,2}=X_{1} \times \mathbb{C}^{n_{2}}=X \times \mathbb{C}^{n_{1}+n_{2}}$ (algebraic equivalence). The homotopy $\eta_{t}^{1}$ lifts to a homotopy $\eta_{t}^{2}:\left.V \rightarrow E^{m, 2}\right|_{V}$, with $\eta_{0}^{2}$ the zero section, and we have a new spray map $s^{2}: E^{m, 2} \rightarrow Z$ satisfying $s^{2}\left(\eta_{t}^{2}\right)=f_{t}$ for all $t \in[0,1]$.

Continuing inductively we obtain after $m$ steps a lifting of the homotopy $\eta_{t}$ to a homotopy $\eta_{t}^{m}:\left.V \rightarrow E^{m, m}\right|_{V}=V \times \mathbb{C}^{N}(t \in[0,1])$, consisting of holomorphic sections of $E^{m, m}=X \times \mathbb{C}^{N}\left(N=n_{1}+n_{2}+\cdots+n_{m}\right)$ over the open subset $V \subset X$, with $\eta_{0}^{m}$ being the zero section. By construction there is an algebraic spray $s^{m}: E^{m, m} \rightarrow Z$ such that $s^{m}\left(\eta_{t}^{m}\right)=f_{t}:\left.V \rightarrow Z\right|_{V}$ for all $t \in[0,1]$.

Recall that $X$ is a closed algebraic submanifold of an affine space $\mathbb{C}^{n}$. The $\mathcal{H}(X)$-convex set $K \subset X$ is then polynomially convex in $\mathbb{C}^{n}$, and $K \times[0,1]$ is polynomially convex in $\mathbb{C}^{n+1}$. (We have identified the segment $[0,1] \subset \mathbb{R}$ with its image in $\mathbb{C}$.) By a small extension of the Oka-Weil theorem we can approximate the homotopy $\left\{\eta_{t}^{m}\right\}_{t \in[0,1]}$ (which is continuous in $(x, t) \in V \times[0,1]$ and holomorphic with respect to $x \in V$ for every fixed $t \in[0,1])$ uniformly on the set $K \times[0,1]$ by a holomorphic polynomial map $\mathbb{C}^{n} \times \mathbb{C} \rightarrow \mathbb{C}^{n} \times \mathbb{C}^{N}$ of the form $\widetilde{g}(x, t)=(x, g(x, t))$, with $g(x, 0)=0$ for $x \in \mathbb{C}^{n}$. By projecting the point $\widetilde{g}(x, t) \in E^{m, m}=X \times \mathbb{C}^{N}$ $(x \in X, t \in \mathbb{C})$ back to the bundle $E^{\prime}=E^{m, 0}$ we obtain an algebraic map $\eta^{\prime}(x, t)$ satisfying lemma 3.6

If $s^{\prime}, \eta^{\prime}$ and $\delta$ are as in lemmas 3.5 and 3.6 with $\delta>0$ chosen sufficiently small, then the algebraic map

$$
F(x, t)=s^{\prime}\left(\eta^{\prime}(x, t)\right) \in Z, \quad(x, t) \in X \times \mathbb{C}
$$

satisfies the conclusion of theorem 3.3

\section{Transversality theOrems FOR holomorphic And Algebraic MAPS}

Given a pair of complex manifolds $X, Y$ we denote by $J^{k}(X, Y)$ the manifold of all $k$-jets of holomorphic maps $X \rightarrow Y$. We have $J^{0}(X, Y)=X \times Y$ and

$$
J^{1}(X, Y)=\left\{(x, y, \lambda): x \in X, y \in Y, \lambda \in \operatorname{Hom}_{\mathbb{C}}\left(T_{x} X, T_{y} Y\right)\right\} .
$$

For a holomorphic map $f: X \rightarrow Y$ and $k \in \mathbb{N}$ we denote by $j^{k} f: X \rightarrow J^{k}(X, Y)$ the $k$-jet extension of $f$; in particular, $j_{x}^{0} f=(x, f(x))$ and $j_{x}^{1} f=\left(x, f(x), d f_{x}\right)$. We shall denote by $\left.j^{k} f\right|_{A}$ the restriction of $j^{k} f$ to points in a subset $A \subset X$.

A stratification of a complex analytic subvariety $A$ (in a complex manifold $X$ ) is a decomposition of $A$ into a locally finite disjoint union of open connected complex manifolds $A_{\alpha}$, called strata of $A$, such that the boundary of each stratum is a union 
of lower dimensional strata (72, p. 227). Whitney proved that every complex analyic subvariety in a complex manifold admits a stratification which is regular with respect to tangent planes ([72], theorem 8.5). We recall how this Condition (a) of Whitney is used in transversality arguments. Given stratified subvarieties $A \subset X, B \subset Y$, let $\mathcal{N} \mathcal{T}_{A, B} \subset J^{1}(X, Y)$ consist of all $(x, y, \lambda) \in J^{1}(X, Y)$ such that, if $x$ belongs to a stratum $A_{\alpha}$ of $A$ and $y$ belongs to a stratum $B_{\beta}$ of $B$ then

$$
\lambda\left(T_{x} A_{\alpha}\right)+T_{y} B_{\beta} \neq T_{y} Y .
$$

(If $x \notin A$ or $y \notin B$ then $(x, y, \lambda) \notin \mathcal{N} \mathcal{T}_{A, B}$.) If the stratifications of $A$ and $B$ satisfy Whitney's Condition (a) then $\mathcal{N} \mathcal{T}_{A, B}$ is closed in $J^{1}(X, Y)$ ([70; 32], p. 38). The set $\mathcal{N} \mathcal{T}_{A, B}$ is also closed in $J^{1}(X, Y)$ if $B$ is a closed smooth (not necessarily complex) submanifold of $Y$; in such case we consider $B$ itself as the only stratum.

Let $A \subset X$ and $B \subset Y$ be stratified complex subvarieties. Given $f \in \mathcal{H}(X, Y)$, we say that $\left.f\right|_{A}$ is transverse to $B$ if the range of $j^{1} f: X \rightarrow J^{1}(X, Y)$ does not intersect the set $\mathcal{N} \mathcal{T}_{A, B}$. Equivalently,

$$
\left(x \in A_{\alpha}, f(x) \in B_{\beta}\right) \Longrightarrow d f_{x}\left(T_{x} A_{\alpha}\right)+T_{f(x)}\left(B_{\beta}\right)=T_{f(x)} Y .
$$

We shall base our discussion on the following condition, similar to the one introduced by Gromov ([39], p. 71), who indicated its application to transversality theorems $\left(\right.$ 39, p. $\left.73,\left(\mathrm{C}^{\prime}\right)\right)$.

Definition 4.1. Let $X$ and $Y$ be holomorphic (resp. algebraic) manifolds. Holomorphic (resp. algebraic) maps $X \rightarrow Y$ satisfy Condition Ell $_{1}$ if for every holomorphic (resp. algebraic) map $f: X \rightarrow Y$ there is a holomorphic (resp. algebraic) map $F: X \times \mathbb{C}^{N} \rightarrow Y$ for some $N \geq \operatorname{dim} Y$ such that $F(\cdot, 0)=f$ and $F(x, \cdot): \mathbb{C}^{N} \rightarrow Y$ has rank equal to $\operatorname{dim} Y$ at $0 \in \mathbb{C}^{N}$ for every $x \in X$.

For validity of Condition $\mathrm{Ell}_{1}$ see proposition 4.6 below, and also [39], p. 72 .

In the sequel, a Whitney stratification of a complex analytic subvariety will mean a stratification satisfying Whitney's Condition (a).

Theorem 4.2. Let $X$ and $Y$ be complex manifolds, with $X$ Stein. If holomorphic maps $X \rightarrow Y$ satisfy Condition $\mathrm{El}_{1}$ then for every pair of closed, Whitney stratified complex analytic subvarieties $A \subset X, B \subset J^{k}(X, Y)(k=0,1, \ldots)$ the set

$$
\left\{f \in \mathcal{H}(X, Y):\left.j^{k} f\right|_{A} \text { is transverse to } B\right\}
$$

is residual in $\mathcal{H}(X, Y)$. The same holds if $B$ is a smooth closed submanifold of $J^{k}(X, Y)$. For $k=0$ the conclusion holds even if $X$ is not Stein.

In the algebraic category we have the analogous result but only on compact sets in the source manifold:

Theorem 4.3. Let $X$ and $Y$ be algebraic manifolds, with $X$ affine algebraic. If algebraic maps $X \rightarrow Y$ satisfy Condition Ell $_{1}$ then for every compact set $K \subset X$ and Whitney stratified complex subvarieties $A \subset X, B \subset J^{k}(X, Y)$ the set

$$
\left\{f \in \mathcal{O}(X, Y):\left.j^{k} f\right|_{A} \text { is transverse to } B \text { on } A \cap K\right\}
$$

is open and dense in $\mathcal{O}(X, Y)$. The same holds if $B$ is a smooth closed submanifold of $J^{k}(X, Y)$. For $k=0$ the conclusion holds without assuming that $X$ be affine.

Theorems 1.3 and 1.4 in $\S 1$ follow immediately by combining theorems 4.24 .3 and proposition 4.6 below. 
Proof. The proofs of theorems 4.2 and 4.3 are parallel up to the point where the Baire property of the space of $\mathcal{H}(X, Y)$ is invoked; in the algebraic case this leaves us with the weaker statement. We shall follow Abraham's reduction [1 to Sard's theorem [60]; although this is well known (see §1.3.7. in [32] as well as [19], 44]), we include a sketch of proof since we shall need small modifications of the standard arguments in some of the proofs in this section and in $\S 5$.

Whitney's Condition (a) implies the following.

Lemma 4.4. Let $X$ and $Y$ be complex manifolds and let $A \subset X, B \subset J^{k}(X, Y)$ be closed, Whitney stratified complex analytic subvarieties. For every compact subset $K$ of $X$ the set

$$
\mathcal{T}_{A, B, K}=\left\{f \in \mathcal{H}(X, Y):\left.j^{k} f\right|_{A} \text { is transverse to } B \text { on } A \cap K\right\}
$$

is open in $\mathcal{H}(X, Y)$. The analogous result holds if $B$ is a smooth closed submanifold.

Proof. Consider the basic case with $B \subset Y$. Given a map $f: X \rightarrow Y$ and a compact set $K \subset X$, the restriction $\left.f\right|_{A}: A \rightarrow B$ is transverse to $B$ at each point of $A \cap K$ if and only if $\left(j^{1} f\right)(K) \cap \mathcal{N} \mathcal{T}_{A, B}=\emptyset$. Assuming this to be the case, and taking into account that $\mathcal{N} \mathcal{T}_{A, B}$ is closed in $J^{k}(X, Y)$ by Whitney's condition, there is a compact set $L \subset X$, with $K \subset \operatorname{Int} L$, such that $\left(j^{1} f\right)(L) \cap \mathcal{N} \mathcal{T}_{A, B}=\emptyset$. If $g \in \mathcal{H}(X, Y)$ is sufficiently uniformly close to $f$ on $L$ then $j^{1} g$ is close to $j^{1} f$ on $K$ and hence $\left(j^{1} g\right)(K) \cap \mathcal{N} \mathcal{T}_{A, B}=\emptyset$. In the general case one applies the same argument with $f$ replaced by the map $j^{k} f: X \rightarrow \widetilde{Y}=J^{k}(X, Y)$.

To prove theorem 4.2 it suffices to show that for every compact $K$ in $X$ the set $\mathcal{T}_{A, B, K} \subset \mathcal{H}(X, Y)$ (which is open in $\mathcal{H}(X, Y)$ by lemma 4.4) is everywhere dense in $\mathcal{H}(X, Y)$. Since $\mathcal{H}(X, Y)$ is a Baire space, the conclusion of theorem 4.2 then follows by taking the intersection of such sets over a countable family of compacts exhausting $X$. In the algebraic case we omit the last step.

Consider first the basic case with $A=X$ and $B \subset Y$. Let $f: X \rightarrow Y$ be a holomorphic (resp. algebraic) map. Choose a map $F: X \times \mathbb{C}^{N} \rightarrow Y$ as in definition 4.1 of Condition $\mathrm{Ell}_{1}$. Let $\pi: X \times \mathbb{C}^{N} \rightarrow \mathbb{C}^{N}$ denote the projection $\pi(x, t)=t$. Fix a compact set $K$ in $X$. Since $\partial_{t} F(x, 0): T_{0} \mathbb{C}^{N} \rightarrow T_{f(x)} Y$ is surjective for every $x \in X$, there are a small ball $D \subset \mathbb{C}^{N}$ around the origin and an open set $U \subset X$ containing $K$ such that $F$ is a submersion of $V=U \times D$ to $Y$. Hence $B^{\prime}=F^{-1}(B) \cap V$ is a closed, Whitney stratified, complex analytic subvariety of $V$ (the strata $B_{\beta}$ of $B$ pull back by $\left.F\right|_{V}$ to the strata $B_{\beta}^{\prime}$ of $\left.B^{\prime}\right)$. Set $f_{t}=F(\cdot, t): X \rightarrow Y$ for $t \in \mathbb{C}^{N}$. If $(x, t) \in B_{\beta}^{\prime}$ then $y=f_{t}(x) \in B_{\beta}$, and by inspecting the definitions we see that the following are equivalent (compare 32, p. 40):

(a) $\left(d f_{t}\right)_{x}\left(T_{x} X\right)+T_{y} B_{\beta}=T_{y} Y$;

(b) $(x, t)$ is a regular point of the restricted projection $\pi: B_{\beta}^{\prime} \rightarrow D$.

By Sard's theorem [60, applied inductively to the components of a projection $\pi$ in (b), we see that the set of regular values of all projections in (b) is residual in $D$. Choosing $t$ in this set and close to 0 we get a map $f_{t}: X \rightarrow Y$ which is transverse to $B$ on $U$ and which approximates $f=f_{0}$ uniformly on $K$. The same argument applies if $B$ is a smooth closed submanifold of $Y$.

If $A$ is a Whitney stratified complex subvariety of $X$, one applies the above argument with $U$ replaced by $U \cap A_{\alpha}$ for a fixed stratum $A_{\alpha}$ of $A$ ( $f$ and $F$ are still 
defined globally on $X)$. This gives a residual set of $t$ 's in $D \subset \mathbb{C}^{N}$ for which $\left.f_{t}\right|_{A_{\alpha} \cap U}$ is transverse to a stratum $B_{\beta}$. Since $A$ and $B$ have at most countably many strata and $\mathbb{C}^{N}$ is a Baire space, we find $t \in \mathbb{C}^{N}$ arbitrarily close to 0 such that $\left.f_{t}\right|_{A \cap U}$ is transverse to $B$.

This proves the basic transversality theorem for holomorphic maps $X \rightarrow Y$. All steps hold for algebraic maps as well, even if the subvarieties $A$ and $B$ are non-algebraic, and we did not need any special properties of $X$ and $Y$ other than Condition Ell $_{1}$ for algebraic maps $X \rightarrow Y$.

To prove theorems 4.2 (resp. 4.3) for $k=0$ we must consider transversality of holomorphic (resp. algebraic) sections $x \rightarrow j_{x}^{1} f=(x, f(x))(x \in X)$ of the trivial fibration $X \times Y \rightarrow X$ with respect to complex (resp. algebraic) subvarieties of $X \times Y$. This is done by obvious modifications of the above arguments, using the fact that the map $(x, t) \rightarrow(x, F(x, t)) \in X \times Y$, constructed above, is a submersion on the subset $U \times D \subset X \times \mathbb{C}^{N}$.

Consider now the case $k>0$. Fix a map $f: X \rightarrow Y$ and a compact set $K \subset X$. The goal is to prove that $f$ can be approximated uniformly on $K$ by holomorphic (resp. algebraic) maps $X \rightarrow Y$ whose $k$-jet extension $j^{k} f$ is such that $\left.j^{k} f\right|_{A}: A \rightarrow$ $J^{k}(X, Y)$ is transverse to the subvariety $B \subset J^{k}(X, Y)$ at each point of $A \cap K$.

Let $F: X \times \mathbb{C}^{N} \rightarrow Y$ be as in definition 4.1 with $F(\cdot, 0)=f$. Recall that $X$ is assumed to be Stein (in theorem 4.2) resp. affine algebraic (in theorem 4.3). Thus we may assume that $X$ is a closed holomorphic (resp. algebraic) submanifold of a Euclidean space $\mathbb{C}^{n}$. Let $\mathcal{W}$ denote the complex vector space of all polynomial maps $P: \mathbb{C}^{n} \rightarrow \mathbb{C}^{N}$ of degree $\leq k$. Consider the holomorphic (resp. algebraic) map $G: X \times \mathcal{W} \rightarrow Y$ defined by

$$
G(x, P)=F(x, P(x)), \quad x \in X, P \in \mathcal{W} .
$$

For each $P \in \mathcal{W}$ set $G_{P}=G(\cdot, P): X \rightarrow Y$; then $G_{0}(x)=F(x, 0)=f(x)$.

Lemma 4.5. The map $\Phi: X \times \mathcal{W} \rightarrow J^{k}(X, Y)$, defined by $\Phi(x, P)=j_{x}^{k}\left(G_{P}\right)$, is a submersion in an open neighborhood of $X \times\{0\}$ in $X \times \mathcal{W}$.

Proof. The argument is local and hence we may assume that $X=\mathbb{C}^{n}$. Write $P=\left(P_{1}, \ldots, P_{N}\right) \in \mathcal{W}$, and let $t=\left(t_{1}, \ldots, t_{N}\right)$ be coordinates on $\mathbb{C}^{N}$. For every multiindex $I=\left(i_{1}, \ldots, i_{n}\right)$ we have

$$
\partial_{x}^{I}\left(G_{P}\right)=\sum_{j=1}^{N} \frac{\partial}{\partial t_{j}} F(x, P(x)) \partial_{x}^{I} P_{j}(x)+H_{I}(x)
$$

where $H_{I}(x)$ contains only terms $\partial_{x}^{J} P$, with $|J|<|I|$, multiplied by various partial derivatives of $F$. Hence the $k$-jet map $j_{x}^{k}\left(G_{P}\right)$ is lower triangular with respect to the components of $j_{x}^{k} P$, and the diagonal terms are nondegenerate at $P=0$ (since $G_{0}(x)=F(x, 0)$ and $\partial_{t} F(x, 0)$ is nondenegenerate), thus proving the lemma.

Sard's theorem, applied to the map $\Phi$ in lemma 4.5 shows that for most $P \in \mathcal{W}$ the map $\left.j^{k}\left(G_{P}\right)\right|_{A}$ is transverse to the subvariety $B \subset J^{k}(X, Y)$ at every point of $A \cap K$. This proves theorems 4.2 and 4.3 .

Combining theorems 4.2 and 4.3 with the following proposition gives several transversality theorems, including those announced in $\$ 1$. Compare with the examples in 39], p. 72 . 
Proposition 4.6. Let $X$ and $Y$ be complex manifolds.

(a) If $Y$ admits a dominating spray $s: Y \times \mathbb{C}^{N} \rightarrow Y$ defined on a trivial bundle then holomorphic maps $X \rightarrow Y$ satisfy Condition $\mathrm{Ell}_{1}$. This holds if $Y$ is a complex homogeneous space.

(b) Let $X$ be Stein. If $Y$ is subelliptic (definition 2.1) or, more generally, if $Y$ enjoys CAP then holomorphic maps $X \rightarrow Y$ satisfy Condition Ell $_{1}$.

(c) If $X$ and $Y$ are algebraic and if $Y$ admits a dominating algebraic spray $s: Y \times \mathbb{C}^{N} \rightarrow Y$ then algebraic maps $X \rightarrow Y$ satisfy Condition Ell $_{1}$.

(d) If $X$ is affine algebraic and $Y$ is algebraically subelliptic then algebraic maps $X \rightarrow Y$ satisfy Condition Ell $_{1}$.

Proof. Fix a holomorphic map $f: X \rightarrow Y$. If $Y$ admits a dominating spray $(E, p, s)$ then $f^{*} E \rightarrow X$ is a holomorphic vector bundle, and there is a fiberwise bijective holomorphic map $\iota: f^{*} E \rightarrow E$ covering $f$. The map $F=s \circ \iota: f^{*} E \rightarrow Y$ satisfies Condition $\operatorname{Ell}_{1}$ for $f$, except that $f^{*} E$ need not be a trivial bundle over $X$. In case (a) the bundle $E \rightarrow Y$ is assumed to be trivial, hence $f^{*} E$ is also trivial and (a) follows. If $Y$ is a homogeneous space of a complex Lie group $G$, with Lie algebra $\mathbf{g}=T_{e} G$, the map $s: Y \times \mathbf{g} \rightarrow Y, s(y, v)=\exp (v) \cdot y$, is a dominating spray defined on a trivial bundle. The analogous argument proves (c).

If $X$ is Stein then by Cartan's Theorem A the holomorphic vector bundle $f^{*} E \rightarrow$ $X$ is generated by finitely many (say $N$ ) holomorphic sections, and hence there is a surjective complex vector bundle map $\tau: X \times \mathbb{C}^{N} \rightarrow f^{*} E$. The map $F=$ $s \circ \iota \circ \tau: X \times \mathbb{C}^{N} \rightarrow Y$ satisfies Condition Ell $_{1}$ with respect to $f=F(\cdot, 0)$. The analogous argument holds in the algebraic case by appealing to Serre's Theorem A (62], p. 237, Théorème 2), thus proving (b) (resp. (d)) for (algebraically) elliptic target manifolds.

Assume now that $Y$ is (algebraically) subelliptic and let $X$ be an affine manifold (Stein resp. affine algebraic). Let $\left(E_{j}, p_{j}, s_{j}\right)$ for $j=1, \ldots, k$ be a finite dominating family of holomorphic (resp. algebraic) sprays on $Y$ (def. 2.1). We shall perform the above procedure several times. In essence we use dominating composed sprays as in the proof of theorem 3.3 above; see also [40, $\$ 1.3 . \mathrm{A}^{\prime}$, and [28], corollary 3.5.

Let $E_{1}^{\prime}=f^{*} E_{1} \rightarrow X$ be the pull-back of $\pi_{1}: E_{1} \rightarrow Y$ by $f: X \rightarrow Y$, and define $\sigma_{1}: E_{1}^{\prime} \rightarrow Y$ by $\sigma_{1}(x, e)=s_{1}(f(x), e)$. As before, there is a surjective complex vector bundle map $X \times \mathbb{C}^{n_{1}} \rightarrow E_{1}^{\prime}$ for some $n_{1} \in \mathbb{N}$. By composing it with $\sigma_{1}$ we obtain a map $f_{1}: X_{1}=X \times \mathbb{C}^{n_{1}} \rightarrow Y$ satisfying $f_{1}(x, 0)=f(x)=y \in Y$ and

$$
\left.\partial_{t} f_{1}(x, t)\right|_{t=0}\left(T_{0} \mathbb{C}^{n_{1}}\right)=\left(d s_{1}\right)_{y}\left(E_{1, y}\right) \subset T_{y} Y \text {. }
$$

Repeating the construction with $f_{1}: X_{1} \rightarrow Y$ and the second spray $s_{2}: E_{2} \rightarrow Y$ we find an integer $n_{2} \in \mathbb{N}$ and a holomorphic map $f_{2}: X_{2}=X_{1} \times \mathbb{C}^{n_{2}}=X \times \mathbb{C}^{n_{1}} \times$ $\mathbb{C}^{n_{2}} \rightarrow Y$ satisfying $f_{2}(x, t, 0)=f_{1}(x, t)$ (hence $\left.f_{2}(x, 0,0)=f(x)=y\right)$ and

$$
\left.\partial_{u} f_{2}(x, 0, u)\right|_{u=0}\left(T_{0} \mathbb{C}^{n_{2}}\right)=\left(d s_{2}\right)_{y}\left(E_{2, y}\right) \subset T_{y} Y .
$$

After $k$ steps we obtain a map $F: X \times \mathbb{C}^{N} \rightarrow Y\left(N=n_{1}+\cdots+n_{k}\right)$ satisfying the following for every $x \in X$ and $y=f(x) \in Y$ :

$$
F(x, 0)=f(x),\left.\quad \partial_{t} F(x, t)\right|_{t=0}\left(T_{0} \mathbb{C}^{N}\right)=\sum_{j=1}^{k}\left(d s_{j}\right)_{y}\left(E_{j, y}\right)=T_{y} Y .
$$


The last equality is the domination property (2.1). This completes the proof of (b) for a subelliptic $Y$; the same proof applies in the algebraic case (d) by appealing to the Theorem A of Serre [62] when passing at each step to a trivial bundle.

It remains to prove (b) when $X$ is Stein and $Y$ enjoys CAP (which is equivalent to the Oka property by corollary [1.7). Let $f: X \rightarrow Y$ be a holomorphic map. Consider the associated embedding $x \in X \rightarrow(x, f(x)) \in X \times Y$ with normal bundle $E=f^{*} T Y \rightarrow X$. By the Docquier-Grauert theorem 13 there are an open neighborhood $V \subset E$ of the zero section $X \subset E$ and a biholomorphic map $G: V \rightarrow G(V) \subset X \times Y$ of the form $G(x, \xi)=(x, g(x, \xi))\left(x \in X, \xi \in E_{x}\right)$ satisfying $g\left(x, 0_{x}\right)=f(x)$. We can extend $g$ to a continuous map $E \rightarrow Y$ without changing its values on a smaller neighborhood of the zero section $X \subset E$.

Since $Y$ enjoys CAP, it also enjoys the Oka property with jet interpolation (corollary 1.4 in [27; see also remark [1.9] above). As $E$ is a Stein manifold, this gives a holomorphic map $\widetilde{g}: E \rightarrow Y$ which agrees with $g$ to the second order along the zero section $X \subset E$. Let $\iota: X \times \mathbb{C}^{N} \rightarrow E$ be a surjective holomorphic vector bundle map (which exists for large $N$ by Cartan's Theorem A). The composition $F=\widetilde{g} \circ \iota: X \times \mathbb{C}^{N} \rightarrow Y$ then satisfies definition 4.1 with respect to the map $f$.

Corollary 4.7. Holomorphic maps from any complex manifold $X$ to a complex homogeneous manifold $Y$ satisfy the basic transversality theorem (for zero-jets). The same holds if $Y=\mathbb{C}^{n} \backslash A$ where $A$ is a thin algebraic subvariety, and in this case the basic transversality theorem also holds for algebraic maps from any algebraic manifold to $\mathbb{C}^{n} \backslash A$.

In the case $Y=\mathbb{P}_{n}$ we recover Bertini's theorem ([32], p. 150).

In 44] Kaliman and Zaidenberg proved the following theorem in which there is no restriction on the target manifold, but the domain of the map shrinks.

Theorem 4.8. (44) Assume that $X$ is a Stein manifold, $Y$ is a complex manifold and $A \subset X, B \subset J^{k}(X, Y)$ are closed, Whitney stratified complex subvarieties. For any $f \in \mathcal{H}(X, Y)$ and any compact set $K \subset X$ there is a holomorphic map $g: U \rightarrow Y$ in an open neighborhood of $K$ such that $\left.j^{k} g\right|_{A \cap U}$ is transverse to $B$, and $g$ approximates $f$ as close as desired uniformly on $K$.

Theorem 4.8 also follows from our proof of theorem 4.2 by using a holomorphic map $F: U \times D \rightarrow Y$ satisfying Condition $\mathrm{El}_{1}$ along $U \times\{0\}$, where $U \subset X$ is an open neighborhood of $K$ and $D \subset \mathbb{C}^{N}$ is a small ball around $0 \in \mathbb{C}^{N}$. Such $F$ exists provided that $K$ admits a basis of open Stein neighborhoods in $X$ (which is the case if $K$ is $\mathcal{H}(X)$-convex). Indeed, the set $\{(x, f(x)): x \in K\} \subset X \times Y$ has an open Stein neighborhood $\Omega \subset X \times Y$ 64, 9], and hence there exist holomorphic vector fields $V^{1}, \ldots, V^{N}$ in $\Omega$ tangent to the fibers of the projection $X \times Y \rightarrow X$ and generating the tangent space of the fiber at each point. Let $\theta_{t}^{j}$ denote the flow of $V^{j}$. For $x \in X$ near $K$ and small $t_{1}, \ldots, t_{N} \in \mathbb{C}$ the map $F\left(x, t_{1}, \ldots, t_{N}\right)=$ $\pi_{Y} \circ \theta_{t_{1}}^{1} \circ \cdots \circ \theta_{t_{N}}^{N}(x, f(x))$ satisfies the required property.

Alternative proof of theorem 1.4 Assume that $X$ is Stein and $Y$ enjoys the Oka property. Let $f: X \rightarrow Y$ be a holomorphic map. Choose compact $\mathcal{H}(X)$-convex subsets $K, L \subset X$ with $K \subset \operatorname{Int} L$. By theorem 4.8 we can approximate $f$ uniformly on $L$ by a holomorphic map $g: U \rightarrow Y$ on an open set $U \supset L$ such that $\left.j^{k} g\right|_{U \cap A}$ is transverse to $B$. If the approximation is sufficiently close, there is a smooth 
map $\widetilde{g}: X \rightarrow Y$ which agrees with $g$ in a neighborhood of $L$, and agrees with $f$ on $X \backslash U$. By the Oka property of $Y$ the map $\widetilde{g}$ can be approximated uniformly on $L$ by holomorphic maps $\widetilde{f}: X \rightarrow Y$. If the approximation is sufficiently close then $\widetilde{f}$ still satisfies the desired transversality condition on $K$ by lemma 4.4 This shows the density of transverse maps on compacts in $X$, thus completing the proof.

We now give an interpolation version of theorem 1.4 Given a closed complex subvariety $X_{0}$ of $X, f_{0} \in \mathcal{H}(X, Y)$ and $r \in\{0,1, \ldots\}$, the set

$$
\mathcal{H}\left(X, Y ; X_{0}, f_{0}, r\right)=\left\{f \in \mathcal{H}(X, Y):\left.j^{r} f\right|_{X_{0}}=\left.j^{r} f_{0}\right|_{X_{0}}\right\}
$$

is a closed metric subspace of $\mathcal{H}(X, Y)$, hence a Baire space.

Theorem 4.9. Let $X$ be a Stein manifold and $Y$ a complex manifold enjoying the Oka property. Let $A \subset X$ and $B \subset J^{k}(X, Y)$ be closed, Whitney stratified complex subvarieties. If $f_{0} \in \mathcal{H}(X, Y)$ is such that $\left.j^{k} f_{0}\right|_{A}$ is transverse to $B$ at all points of $A \cap X_{0}$ then for every integer $r \geq k$ there is a residual set of $f \in \mathcal{H}\left(X, Y ; X_{0}, f_{0}, r\right)$ for which $\left.j^{k} f\right|_{A}$ is transverse to $B$.

Proof. Since $r \geq k$, the set of all $f \in \mathcal{H}\left(X, Y ; X_{0}, f_{0}, r\right)$ for which $\left.j^{k} f\right|_{A}$ is transverse to $B$ at all points of $A \cap X_{0}$ is open in $\mathcal{H}\left(X, Y ; X_{0}, f_{0}, r\right)$. To prove theorem 4.9 it thus suffices to show that we can approximate the initial map $f_{0}$ uniformly on any compact $\mathcal{H}(X)$-convex subset $K \subset X$ by $f \in \mathcal{H}\left(X, Y ; X_{0}, f_{0}, r\right)$ such that $\left.j^{k} f\right|_{A}$ is transverse to $B$ at every point of $A \cap K$.

Assume first that holomorphic maps $X \rightarrow Y$ satisfy Condition $\mathrm{Ell}_{1}$, and let $F: X \times \mathbb{C}^{N} \rightarrow Y$ be as in definition 4.1] with $F(\cdot, 0)=f_{0}$. Consider the basic case $k=0, B \subset Y$. There exist functions $g_{1}, \ldots, g_{l} \in \mathcal{H}(X)$ which vanish to order $r+1$ on the subvariety $X_{0}=\left\{x \in X: g_{j}(x)=0, j=1, \ldots, l\right\}$. For every $x \in X$ let $\sigma_{x}:\left(\mathbb{C}^{N}\right)^{l} \rightarrow \mathbb{C}^{N}$ be defined by

$$
\sigma_{x}\left(t_{1}, \ldots, t_{l}\right)=\sum_{j=1}^{l} t_{j} g_{j}(x), \quad t_{j} \in \mathbb{C}^{N}, j=1,2, \ldots, l .
$$

Clearly $\sigma_{x}$ is surjective if $x \in X \backslash X_{0}$ and is the zero map if $x \in X_{0}$. The map $\widetilde{F}: X \times \mathbb{C}^{N l} \rightarrow Y$, defined by $\widetilde{F}(x, t)=\widetilde{F}\left(x, t_{1}, \ldots, t_{l}\right)=F\left(x, \sigma_{x}\left(t_{1}, \ldots, t_{l}\right)\right)$, is a submersion with respect to $t$ (at $t=0$ ) if $x \in X \backslash X_{0}$, and is degenerate (constant) if $x \in X_{0}$. Hence the proof of theorem 4.2 applies over $X \backslash X_{0}$.

Let $f_{t}=\widetilde{F}(\cdot, t): X \rightarrow Y$ for $t \in \mathbb{C}^{N l}$. By construction $\left.j^{r} f_{t}\right|_{X_{0}}=\left.j^{r} f_{0}\right|_{X_{0}}$ for every $t$. Choose a compact set $K \subset X$. By the assumption $\left.f_{0}\right|_{A}$ is transverse to $B$ on $A \cap X_{0}$. Hence there is an open neighborhood $U \subset X$ of $A \cap X_{0} \cap K$ such that $\left.f_{t}\right|_{A \cap U}$ is transverse to $B$ for every $t$ sufficiently close to 0 (lemma 4.4). The set $K^{\prime}=K \backslash U \subset X \backslash X_{0}$ is compact and hence for most values of $t$ the map $\left.f_{t}\right|_{A}$ is transverse to $B$ on $A \cap K^{\prime}$. Thus $\left.f_{t}\right|_{A}$ is transverse to $B$ on $K \cap A$ for most $t$ close to 0 which concludes the proof for $k=0$. Similarly one obtains the proof for $k>0$ by following the arguments in the proof of theorem 4.2

The same proof gives a semiglobal version of theorem 4.9] analogous to theorem 4.8 without any restriction on the manifold $Y$.

The proof of the general case is completed exactly as the alternative proof of theorem 1.4 given above, using corollary 1.4 in [27] to the effect that the (basic) Oka property of $Y$ implies the Oka property with jet interpolation on a closed complex subvariety $X_{0}$ of a Stein manifold $X$. 
In the algebraic category the global transversality theorem holds under the following stronger assumption on $Y$.

Proposition 4.10. If $Y$ is an algebraic manifold with a submersive algebraic spray $s: E \rightarrow Y$ (i.e., such that $s: E_{y} \rightarrow Y$ is a submersion for every $y \in Y$ ) then algebraic maps $X \rightarrow Y$ from any affine algebraic manifold $X$ satisfy the jet transversality theorem with respect to closed complex analytic subvarieties.

Proof. Let $f_{0}: X \rightarrow Y$ be an algebraic map. Pulling back the submersive algebraic spray $s: E \rightarrow Y$ by $f_{0}$ we obtain an algebraic submersion $F: X \times \mathbb{C}^{N} \rightarrow Y$ satisfying $f_{0}=F(\cdot, 0)$ (compare with the proof of proposition 4.6). Given closed complex subvarieties $A \subset X$ and $B \subset Y$, Sard's theorem shows that for a generic choice of $t \in \mathbb{C}^{N}$ the algebraic map $\left.f_{t}\right|_{A}=\left.F(\cdot, t)\right|_{A}$ is transverse to $B$ (see the proof of theorem 4.2). Similarly one obtains the jet transversality theorem by considering maps $x \rightarrow F(x, P(x))$ for polynomials $P: \mathbb{C}^{n} \rightarrow \mathbb{C}^{N}$, where $X$ is embedded in $\mathbb{C}^{n}$.

\section{THE HOMOTOPY PRINCIPLE FOR HOLOMORPHIC SUBMERSIONS}

Given complex manifolds $X$ and $Y$, we denote by $\mathcal{S}(X, Y)$ the set of all pairs $(f, \iota)$ where $f: X \rightarrow Y$ is a continuous map and $\iota: T X \rightarrow T Y$ is a fiberwise surjective complex vector bundle map such that the diagram in Fig. 1 commutes.

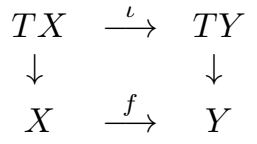

Figure 1: The space $\mathcal{S}(X, Y)$

The existence of $\iota$ in Fig. 1 (for a given $f$ ) is invariant under homotopies of the base map, and $T f=(f, d f) \in \mathcal{S}(X, Y)$ precisely when $f: X \rightarrow Y$ is a holomorphic submersion. (Here $T f$ denotes the tangent map of $f$.) Hence a necessary condition for a continuous map $f: X \rightarrow Y$ to be homotopic to a holomorphic submersion $X \rightarrow Y$ is that $f$ be covered by a map $\iota: T X \rightarrow T Y$ such that $(f, \iota) \in \mathcal{S}(X, Y)$. By theorem II in 23] this condition is also sufficient if $X$ is Stein and $Y=\mathbb{C}^{p}$, $p<\operatorname{dim} X$. Here we prove the same result when the target manifold is of Class $\mathcal{A}$, or a holomorphic quotient of such manifold.

Theorem 5.1. Assume that $Y$ is a complex manifold which admits an unramified holomorphic covering $\widetilde{Y} \rightarrow Y$ by a quasi-projective algebraic manifold $\widetilde{Y}$ of Class $\mathcal{A}$ (def. [2.3). If $X$ is a Stein manifold with $\operatorname{dim} X>\operatorname{dim} Y$ and $K \subset X$ is a compact $\mathcal{H}(X)$-convex subset then the following hold.

(a) For any $(f, \iota) \in \mathcal{S}(X, Y)$ the map $f$ is homotopic to a holomorphic submersion $f_{1}: X \rightarrow Y$. If in addition $\left.f\right|_{K}: K \rightarrow Y$ is a holomorphic submersion and $\left.\iota\right|_{K}=\left.d f\right|_{K}$ then $f_{1}$ can be chosen to approximate $f$ uniformly on $K$.

(b) Holomorphic submersions $f_{0}, f_{1}: X \rightarrow Y$ are regularly homotopic through a family of holomorphic submersions $X \rightarrow Y$ if and only if their tangent maps $T f_{0}$ and $T f_{1}$ belong to the same path connected component of $\mathcal{S}(X, Y)$. 
(c) If $\operatorname{dim} X \geq 2 \operatorname{dim} Y-1$ then every continuous map $X \rightarrow Y$ is homotopic to a holomorphic submersion; if $\operatorname{dim} X \geq 2 \operatorname{dim} Y$ then any two holomorphic submersions $X \rightarrow Y$ are regularly homotopic.

Theorem 5.1 implies results on the existence of nonsingular holomorphic foliations of the source manifold $X$; see [23] for the case $Y=\mathbb{C}^{p}$.

Example 5.2. We list some examples of (holomorphic quotients of) quasi-projective algebraic manifolds of Class $\mathcal{A}$ to which theorem [5.1] applies.

(1) $Y=\widehat{Y} \backslash A$ where $\widehat{Y}$ is an affine space, a projective space or a Grassmanian and $A$ is a thin algebraic subvariety of $\widehat{Y}$.

(2) $Y=\mathbb{C}^{p} / \Gamma$ where $\Gamma$ is a lattice in $\mathbb{C}^{p}$. This class includes all complex tori.

(3) Hopf manifolds are quotients of $\mathbb{C}_{*}^{p} \stackrel{\text { def }}{=} \mathbb{C}^{p} \backslash\{0\}(p \geq 2)$ by an infinite cyclic group, or a finite extension of such group ([2], p. 225).

(4) Let $\pi: W \rightarrow Y$ be a holomorphic fiber bundle whose base $Y$ is a quotient of a Class $\mathcal{A}_{0}$ manifold, the fiber $\pi^{-1}(y)$ is $\mathbb{C}^{m}$ respectively $\mathbb{P}_{m}$, and the structure group is $G L_{m}(\mathbb{C})$ respectively $P G L_{m}(\mathbb{C})$. It is easily seen that $W$ is then a quotient of a Class $\mathcal{A}_{0}$ manifold.

Part (c) of theorem 5.1 follows from (a) and (b) by topological reasons (see corollary 2.3 in 24]). We shall reduce parts (a) and (b) to theorem 2.1 in 24. To this end we must recall from 24] a certain holomorphic flexibility property, called Property $S_{n}$, which is the localization to Euclidean spaces of the homotopy principle for holomorphic submersions from $n$-dimensional Stein manifolds to $Y$.

Let $z=\left(z_{1}, \ldots, z_{n}\right), z_{j}=x_{j}+i y_{j}$, denote the coordinates on $\mathbb{C}^{n}$. Set

$$
P=\left\{z \in \mathbb{C}^{n}:\left|x_{j}\right| \leq 1,\left|y_{j}\right| \leq 1, j=1, \ldots, n\right\} .
$$

A compact convex subset $K \subset \mathbb{C}^{n}$ is special if

$$
K=\left\{z \in P: y_{n} \leq h\left(z_{1}, \ldots, z_{n-1}, x_{n}\right)\right\}
$$

where $h$ is a smooth concave function with values in $(-1,+1)$.

Definition 5.3. Let $Y$ be a complex manifold and $d$ a distance function on $Y$ induced by a Riemannian metric.

(a) $Y$ satisfies Property $\mathrm{S}_{\mathrm{n}}$ if for every holomorphic submersion $f: K \rightarrow Y$ on a special compact convex set $K(5.2)$ and for every $\epsilon>0$ there is a holomorphic submersion $\widetilde{f}: P \rightarrow Y$ satisfying $\sup _{x \in K} d(f(x), \widetilde{f}(x))<\epsilon$.

(b) $Y$ satisfies Property $\mathrm{HS}_{\mathrm{n}}$ if for every homotopy of holomorphic submersions $f_{t}: K \rightarrow Y(t \in[0,1])$ such that $f_{0}$ and $f_{1}$ extend to holomorphic submersions $P \rightarrow Y$ there is for every $\epsilon>0$ a homotopy of holomorphic submersions $\widetilde{f}_{t}: P \rightarrow Y(t \in[0,1])$ satisfying $\widetilde{f}_{0}=f_{0}, \widetilde{f}_{1}=f_{1}$, and $\sup \left\{d\left(f_{t}(x), \widetilde{f}_{t}(x)\right): x \in K, t \in[0,1]\right\}<\epsilon$.

According to theorem 2.1 in 24], the conclusion of (a) (respectively of (b)) in theorem 5.1 holds provided that $Y$ satisfies Property $\mathrm{S}_{\mathrm{n}}$ (respectively Property $\mathrm{HS}_{\mathrm{n}}$ ) with $n=\operatorname{dim} X$. Furthermore, both properties are obviously invariant when passing to an unramified holomorphic covering or quotient. Hence theorem [5.1] is implied by the following proposition. 
Proposition 5.4. A quasi-projective algebraic manifold $Y$ of Class $\mathcal{A}$ (def. 2.3) satisfies Properties $\mathrm{S}_{\mathrm{n}}$ and $\mathrm{HS}_{\mathrm{n}}$ for every $n>\operatorname{dim} Y$.

Proof. Let $Y$ be a manifold of class $\mathcal{A}$. We may (and shall) assume that $Y$ is connected. We first prove Property $\mathrm{S}_{\mathrm{n}}$. Let $f: K \rightarrow Y$ be a holomorphic submersion from an open neighborhood of a special compact convex $K \subset \mathbb{C}^{n}(5.2)$. By corollary 2.4 the manifold $Y$ is algebraically subelliptic, and corollary 3.2 then shows that $f$ can be approximated uniformly on $K$ by algebraic maps $\mathbb{C}^{n} \rightarrow Y$. Thus it suffices to consider the case when $f: \mathbb{C}^{n} \rightarrow Y$ is an algebraic map which is a submersion at every point of $K$.

We denote by $\Sigma \subset \mathbb{C}^{n}$ the ramification locus of $f$ (the set of nonsubmersion points). This is an algebraic subvariety of $\mathbb{C}^{n}$ which does not intersect $K$.

Case 1: $\operatorname{dim} \Sigma \leq n-2$. Lemma 3.4 in 23 provides a holomorphic automorphism $\psi$ of $\mathbb{C}^{n}$ which approximates the identity map in a neighborhood of $K$ and satisfies $\psi(P) \cap \Sigma=\emptyset$. The map $\tilde{f}=f \circ \psi: P \rightarrow Y$ is a holomorphic submersion approximating $f$ on $K$, thus proving Property $\mathrm{S}_{\mathrm{n}}$. For this argument it suffices to assume that $f$ is a holomorphic submersion (with values in $Y$ ) defined in the complement of a thin algebraic subvariety in $\mathbb{C}^{n}$.

Case 2: $\operatorname{dim} \Sigma=n-1$. We shall reduce to Case 1 by inductively removing all $(n-1)$-dimensional irreducible components from the ramification locus $\Sigma$, changing the map at every step.

Choose an irreducible component $\Sigma^{\prime} \subset \Sigma$ of dimension $n-1$ and a point $z_{0} \in \Sigma^{\prime}$ which does not belong to any other irreducible component of $\Sigma$. By definition 2.3 we have $Y=\widehat{Y} \backslash A$ where $\widehat{Y}$ is a connected manifold of Class $\mathcal{A}_{0}$ and $A$ is a thin algebraic subvariety of $\widehat{Y}$. Let $U \subset \widehat{Y}$ be a Zariski open set isomorphic to $\mathbb{C}^{p}$ and containing the point $y_{0}=f\left(z_{0}\right)$. Let $s_{0}: U \times \mathbb{C}^{p} \rightarrow U \simeq \mathbb{C}^{p}$ denote the spray $s_{0}(y, t)=y+t$. Choose a closed algebraic subvariety $Y_{0} \subset \widehat{Y}$ of pure dimension $p-1$ such that $\widehat{Y}=Y_{0} \cup U$ and $y_{0} \notin Y_{0}$. Let $L=\left[Y_{0}\right]^{-1}$ where $\left[Y_{0}\right] \rightarrow \widehat{Y}$ is the holomorphic line bundle defined by the divisor of $Y_{0}$. Let $\tau_{p}=\widehat{Y} \times \mathbb{C}^{p}$.

By proposition 1.3 in [22, p. 541 (or §3.5.B. and §3.5.C. in [40]) there are an integer $m \in \mathbb{N}$ and an algebraic spray $s: E=\tau_{p} \otimes L^{\otimes m} \rightarrow \widehat{Y}$ such that $s(y, t)=y$ for all $y \in Y_{0}$ and $t \in E_{y}$, and $s$ equals $s_{0}$ over the open set $\widehat{Y} \backslash Y_{0} \subset U$, using an identification of $\left.E\right|_{U}$ with $\left.\tau_{p}\right|_{U}$. (The line bundle $L$ is trivial over $\widehat{Y} \backslash Y_{0}$.)

By Serre's Theorem A 62 the algebraic vector bundle $f^{*}(E) \rightarrow \mathbb{C}^{n}$ is generated by finitely many (say $q$ ) algebraic sections, and hence there is a surjective algebraic vector bundle map $\rho: \mathbb{C}^{n} \times \mathbb{C}^{q} \rightarrow f^{*} E$. Let $\iota: f^{*} E \rightarrow E$ be the natural map covering $f$. Set $Z=f^{-1}\left(Y_{0}\right) \subset \mathbb{C}^{n}$. The algebraic map $F=s \circ \iota \circ \rho: \mathbb{C}^{n} \times \mathbb{C}^{q} \rightarrow \widehat{Y}$ satisfies the following properties:

(a) $F(z, 0)=f(z)\left(z \in \mathbb{C}^{n}\right)$,

(b) $F(z, t)=f(z)\left(z \in Z \subset \mathbb{C}^{n}, t \in \mathbb{C}^{q}\right)$, and

(c) $F(z, \cdot): \mathbb{C}^{q} \rightarrow \widehat{Y}$ is a submersion for every point $z \in V:=\mathbb{C}^{n} \backslash Z$.

The proof of theorem 4.2 (see especially lemma 4.5 and proposition 4.10) gives a polynomial map $P: \mathbb{C}^{n} \rightarrow \mathbb{C}^{q}$ such that the algebraic map $f_{1}: \mathbb{C}^{n} \rightarrow \widehat{Y}$ defined by $f_{1}(x)=F(x, P(x))\left(x \in \mathbb{C}^{n}\right)$ satisfies the following properties:

(i) $f_{1}$ approximates $f$ uniformly on $K$ as close as desired, 
(ii) $\left.j^{1} f\right|_{Z}=\left.j^{1} f_{1}\right|_{Z}$,

(iii) $\left.f_{1}\right|_{V}$ is transverse to the subvariety $A \subset \widehat{Y}\left(V=\mathbb{C}^{n} \backslash Z\right)$, and

(iv) the ramification locus of $\left.f_{1}\right|_{V}$ has dimension $\leq n-2$.

To obtain (iv) we choose $P$ such that $j^{1} f_{1}$ is transverse to the subvariety of $J^{1}\left(\mathbb{C}^{n}, Y\right)$ consisting of all jets of non-maximal rank; this subvariety has codimension $n-\operatorname{dim} Y+1 \geq 2$ which gives (iv).

Let $C \subset \mathbb{C}^{n}$ denote the ramification locus of $f_{1}: \mathbb{C}^{n} \rightarrow \widehat{Y}$; thus $\operatorname{dim} C \backslash Z \leq n-2$ by (iv). The set

$$
\Sigma_{1}=(\Sigma \cap Z) \cup f_{1}^{-1}(A) \cup C
$$

is an algebraic subvariety of $\mathbb{C}^{n}$ which does not intersect $K$, provided that the approximation of $f$ by $f_{1}$ is sufficiently close on $K$ (we shrink $K$ a little). The restriction of $f_{1}$ to $\mathbb{C}^{n} \backslash \Sigma_{1}$ maps the latter set submersively to $Y=\widehat{Y} \backslash A$.

We claim that $\Sigma_{1}$ has less $(n-1)$-dimensional irreducible components than $\Sigma$. Observe first that $\operatorname{dim}\left(\Sigma_{1} \backslash Z\right) \leq n-2$ by properties (iii) and (iv) of $f_{1}$. Next we show $\operatorname{dim}\left[(Z \backslash \Sigma) \cap\left(f_{1}^{-1}(A) \cup C\right)\right] \leq n-2$. If $z \in Z \backslash \Sigma$ then $f$ is unramified at $z$ by the definition of $\Sigma$; furthermore, $j_{z}^{1} f_{1}=j_{z}^{1} f$ by property (ii), and hence $f_{1}$ is also unramified at such point $z$, thus showing $(Z \backslash \Sigma) \cap C=\emptyset$. This also implies that $\left.f_{1}\right|_{Z \backslash \Sigma}$ is transverse to $A$, whence $\operatorname{dim} f_{1}^{-1}(A) \cap(Z \backslash \Sigma) \leq n-2$. Hence the $(n-1)$-dimensional irreducible components of $Z_{1}$ are the same as those of $\Sigma \cap Z$. Since $z_{0} \in \Sigma^{\prime} \backslash Z$, the component $\Sigma^{\prime}$ of $\Sigma$ is not among them which proves the claim.

Repeating the same argument with the pair $\left(f_{1}, \Sigma_{1}\right)$ gives an algebraic map $f_{2}: \mathbb{C}^{n} \rightarrow \widehat{Y}$ and an algebraic subvariety $\Sigma_{2} \subset \mathbb{C}^{n}$ with less $(n-1)$-dimensional components than $\Sigma_{1}$ such that $f_{2}: \mathbb{C}^{n} \backslash \Sigma_{2} \rightarrow Y$ is a submersion which approximates $f_{1}$ (and hence $f$ ) uniformly on $K$. Proceeding inductively we obtain in finitely many steps a holomorphic submersion $\widetilde{f}: \mathbb{C}^{n} \backslash \widetilde{\Sigma} \rightarrow Y$, where $\widetilde{\Sigma}$ is an algebraic subvariety with $\operatorname{dim} \widetilde{\Sigma} \leq n-2$, such that $\left.\widetilde{f}\right|_{K}$ approximates $\left.f\right|_{K}$. We complete the proof as in Case 1. This establishes Property $\mathrm{S}_{\mathrm{n}}$ of $Y$.

It remains to prove Property $\mathrm{HS}_{\mathrm{n}}$ for $n>\operatorname{dim} Y$. We shall need the following lemma on algebraic approximation of the initial homotopy $f_{t}$.

Lemma 5.5. Let $K \subset P \subset \mathbb{C}^{n}$ be as in (5.1), (5.2). Let $f_{t}: K \rightarrow Y$ for $t \in[0,1]$ be a homotopy of holomorphic maps such that $f_{0}, f_{1}$ extend to holomorphic maps $P \rightarrow Y$. Let $d$ be a distance function induced by a Riemannian metric on $Y$. For every $\epsilon>0$ there is an algebraic map $F: \mathbb{C}^{n+1} \rightarrow Y$ such that $d\left(F(x, t), f_{t}(x)\right)<\epsilon$ for all $(x, t) \in(K \times[0,1]) \cup(P \times\{0,1\}) \subset \mathbb{C}^{n} \times \mathbb{C}$.

Proof. By corollary 3.2 we may assume that $f_{0}$ is algebraic. Since $Y$ is subelliptic, theorem 4.5 in [28] implies that we can approximate the homotopy $f_{t}$ uniformly on $K$ by a homotopy consisting of holomorphic maps $\widetilde{f}_{t}: P \rightarrow Y(t \in[0,1])$ such that $\tilde{f}_{t}=f_{t}$ for $t=0$ and $t=1$. Hence we may assume that the original homotopy $f_{t}$ is defined and holomorphic on $P$, and the initial map $f_{0}$ is algebraic. Lemma 5.5 now follows from theorem 3.1 applied with the compact set $P$ in $X=\mathbb{C}^{n}$.

Assume that the map $f_{t}$ in lemma [5.5 is a submersion $K \rightarrow Y$ for $t \in[0,1]$, and it is a submersion $P \rightarrow Y$ for $t=0$ and $t=1$. If the approximation of $f_{t}$ by the algebraic map $F_{t}=F(\cdot, t): \mathbb{C}^{n} \rightarrow Y$, furnished by lemma [5.5] is sufficiently 
uniformly close on the respective sets then we may assume the same properties for $F_{t}$ after a slight shrinking of $K$ and $P$.

Consider now $F$ as a map to $\widehat{Y}$. A transversality argument, analogous to the one used in the proof of Property $\mathrm{S}_{\mathrm{n}}$, gives a nearby algebraic map (still called $F$ ) such that for all points $(x, t) \in \mathbb{C}^{n+1} \backslash \Sigma$ outside of an algebraic subvariety with $\operatorname{dim} \Sigma \leq n-1$ we have $F(x, t) \in Y$ and $\partial_{x} F_{t}: T_{x} \mathbb{C}^{n} \rightarrow T_{F(x, t)} Y$ is surjective.

Fix such $F$. For all but finitely many values of $t \in \mathbb{C}$ the set $\Sigma_{t}=\{x \in$ $\left.\mathbb{C}^{n}:(x, t) \in \Sigma\right\}$ then satisfies $\operatorname{dim} \Sigma_{t} \leq n-2$. By a small smooth deformation $\tau:[0,1] \rightarrow \mathbb{C}$ of the parameter interval $[0,1] \subset \mathbb{R} \subset \mathbb{C}$ inside $\mathbb{C}$ we can avoid this exceptional set of $t$ 's and obtain a homotopy of algebraic submersions $F_{\tau(t)}: \mathbb{C}^{n} \backslash \Sigma_{\tau(t)} \rightarrow Y$ with $\operatorname{dim} \Sigma_{\tau(t)} \leq n-2$ for all $t \in[0,1]$. Since $F_{\tau(t)}$ approximates $f_{t}$ on $K$ (resp. on $P$ for $t=0$ and $t=1$ ), we have $\Sigma_{\tau(t)} \cap K=\emptyset$ for all $t \in[0,1]$, and $\Sigma_{\tau(t)} \cap P=\emptyset$ for $t \in\{0,1\}$. (We shrink $K$ and $P$ slightly.)

Lemma 3.4 in 23 gives a family of holomorphic automorphisms $\psi_{t} \in \operatorname{Aut} \mathbb{C}^{n}$ depending smoothly on $t \in[0,1]$, with $\psi_{0}, \psi_{1}$ being the identity map, such that $\psi_{t}(P) \cap \Sigma_{\tau(t)}=\emptyset$ for every $t \in[0,1]$. The homotopy $\widetilde{f}_{t}=F_{\tau(t)} \circ \psi_{t}: P \rightarrow Y$ $(t \in[0,1])$ consists of holomorphic submersions approximating $f_{t}$ on $K$ (resp. on $P$ for $t \in\{0,1\}$ ). If $\widetilde{f}_{0}$ is sufficiently close to $f_{0}$ on $P$ then we can join the two maps by a homotopy of submersions $P \rightarrow Y$; the same holds at $t=1$.

Remark 5.6. Our proof shows that every algebraic manifold $Y$ with a submersive algebraic spray satisfies Properties $\mathrm{S}_{\mathrm{n}}$ and $\mathrm{HS}_{\mathrm{n}}$ (and hence the conclusion of theorem 5.1) for every $n>\operatorname{dim} Y$.

\section{Flexibility properties of CURVES AND Surfaces}

In this section we survey the holomorphic flexibility properties of complex curves (Riemann surfaces) and complex surfaces. Several of the results mentioned here were first proved in [40, 22] and [26].

6.1. Riemann surface. We have the following precise result. The equivalence of (d), (e) and (f) is well known and is stated only for completeness.

Proposition 6.1. The following are equivalent for a Riemann surface $Y$ :

(a) $Y$ is elliptic (it admits a dominating spray).

(b) $Y$ enjoys the Oka property.

(c) Holomorphic submersions $X \rightarrow Y$ from any Stein manifold $X$ of dimension $\operatorname{dim} X>1$ satisfy the conclusion of theorem 5.1.

(d) $Y$ is dominable by $\mathbb{C}$.

(e) $Y$ is not hyperbolic.

(f) $Y$ is one of the surfaces $\mathbb{P}_{1}, \mathbb{C}, \mathbb{C}^{*}$ or a torus $\mathbb{C} / \Gamma$.

Proof. The universal covering of any Riemann surface $Y$ is one of the surfaces $\mathbb{P}_{1}$, $\mathbb{C}$, or $\mathbb{U}=\{z \in \mathbb{C}:|z|<1\}$. $\mathbb{P}_{1}$ has no nontrivial quotients while $\mathbb{C}$ covers $\mathbb{C}_{*}$ and the tori $\mathbb{C} / \Gamma$. All of these are homogeneous and hence admit a dominating spray, and they also satisfy theorem 5.1 since $\mathbb{P}_{1}$ and $\mathbb{C}$ are of Class $\mathcal{A}$. The disc $\mathbb{U}$ and its quotients are hyperbolic and hence do not satisfy any flexibility property. 
6.2. Compact complex surfaces. Let $Y$ be a compact complex surface of Kodaira dimension $\kappa$ at most one, the latter condition being implied by holomorphic dominability of $Y$ by $\mathbb{C}^{2}$. We first consider the case when $Y$ is projective algebraic. By the Enriques-Kodaira classification (Chapter VI in 2]) every such $Y$ is obtained from one of the following minimal surfaces $X$ by blowing up points:

(6.2.1) A holomorphic $\mathbb{P}_{1}$-bundle over a curve (Riemann surface) $C ; \kappa=-\infty$.

(6.2.2) A torus (a quotient of $\mathbb{C}^{2}$ by a lattice of real rank four); $\kappa=0$.

(6.2.3) A K3 surface (a compact simply connected surface with trivial canonical bundle $\left.K_{X}=\Lambda^{2}\left(T^{*} X\right)\right) ; \kappa=0$.

(6.2.4) A minimal surface $X$ with the structure of an elliptic fibration (2], pp. $200-219) ; \kappa \in\{-\infty, 0,1\}$.

These classes are not entirely disjoint; for example, the moduli space of K3 surfaces contains a dense codimension one subset consisting of elliptic fibrations. All surfaces on the above list are minimal (i.e., not containing any smooth rational $(-1)$-curves), and every compact projective surface is obtained from one of them (or from a minimal surface of general type) by a finite sequence of blow-ups. If $\operatorname{kod} Y \geq 0$ then $Y$ is obtained from a unique minimal $X$.

A complete list of compact (not necessarily projective algebraic) complex surfaces, classified according to the value of Kodaira dimension $\kappa<2$, can be found in 2] (Table 10 on p. 244). Some of these can be included in the classes already listed above; for example, the class of elliptic fibrations (6.2.4) includes all bi-elliptic surfaces and all primary Kodaira surfaces ([2, V.5). Besides these one also has

(6.2.5) Secondary Kodaira surfaces; $\kappa=0$. These are unramified holomorphic quotients of primary Kodaira surfaces.

(6.2.6) Class VII surfaces $\left(\kappa(X)=-\infty, b_{1}(X)=1\right)$. This class includes the Hopf surfaces ([2, V.18), Inoue surfaces (2], V.19.), and several others.

Buzzard and Lu proved in 6] (theorem 1.1) that for a compact projective surface $X$ of Kodaira dimension $\kappa<2$ which is not birational to an elliptic or a Kummer $\mathrm{K} 3$ surface, dominability by $\mathbb{C}^{2}$ is equivalent to the existence of a holomorphic map $\mathbb{C} \rightarrow X$ with Zariski-dense image. For compact surfaces with $\kappa<2$ which are not birationally equivalent to a K3 surface they also gave a characterization of dominability in terms of the fundamental group (theorem 1.2 in [6]). By the same theorem, every compact projective surface which is birationally equivalent to an elliptic or a Kummer K3 surface is dominable by $\mathbb{C}^{2}$. The same holds in the category of compact (non-projective) surfaces (propositions 4.4 and 4.5 in [6]).

In the sequel we consider the question which of the compact surfaces from the above list enjoy the Oka property.

Complex tori (6.2.2), being unramified quotients of $\mathbb{C}^{2}$, satisfy the Oka property and the conclusion of theorem 5.1

A secondary Kodaira surface $X(6.2 .5)$ is covered by a primary Kodaira surface $\widetilde{X}$ which admits the structure of an elliptic fibration; hence $X$ enjoys the Oka property if and only if $\widetilde{X}$ does. There exist surfaces with the structure of a ramified elliptic fibration which are not dominable by $\mathbb{C}^{2}$ (example 6.3 below).

A Hopf surface (a special case of a Class VII surface (6.2.6)) is a quotient $\mathbb{C}_{*}^{2} / \Gamma$ by a finite extension of a cyclic group. It enjoys the Oka property (Corollary 1.5 (i) in [26]) since its universal covering $\mathbb{C}_{*}^{2}=\mathbb{C}^{2} \backslash\{0\}$ is of Class $\mathcal{A}$. 
An Inoue surface (6.2.6) is a quotient of $\mathbb{D} \times \mathbb{C}$ (where $\mathbb{D}$ is the unit disc), and hence is not dominable by $\mathbb{C}^{2}$. These surfaces don't admit any closed complex curves, and any nonconstant image of $\mathbb{C}$ is Zariski dense.

For surfaces (6.2.1) (which include all Hirzebruch surfaces) and for the unramified elliptic fibrations (6.2.4) the following proposition gives a complete answer.

Proposition 6.2. If $C$ is a Riemann surface (not necessarily compact) and $\pi: X \rightarrow$ $C$ is either a holomorphic fiber bundle with fiber $\mathbb{P}_{1}$ or an unramified elliptic fibration without exceptional fibers then the following are equivalent:

(a) $X$ satisfies the Oka property.

(b) $X$ is dominable by $\mathbb{C}^{2}$.

(c) $C$ is not hyperbolic.

Proof. $(\mathrm{a}) \Rightarrow(\mathrm{b})$ holds in general (see $\S 1),(\mathrm{b}) \Rightarrow(\mathrm{c})$ is obvious, and $(\mathrm{c}) \Rightarrow(\mathrm{a})$ follows from corollary 1.6 and proposition 6.1 (in this case $C$ is one of the Riemann surfaces $\mathbb{P}_{1}, \mathbb{C}, \mathbb{C}_{*}$ or a torus $\left.\mathbb{C} / \Gamma\right)$. This proves proposition 6.2 We observe in addition that the total space $X$ of a holomorphic fiber bundle $X \rightarrow C$ with fiber $\mathbb{P}_{1}$ and base $C \in\left\{\mathbb{P}_{1}, \mathbb{C}\right\}$ is of Class $\mathcal{A}$; if $C$ is $\mathbb{C}_{*}$ or a torus $\mathbb{C} / \Gamma$ then $X$ is a quotient of a Class $\mathcal{A}$ manifold. Theorem 5.1 applies to all such manifolds.

For ramified elliptic fibrations $\pi: X \rightarrow C$ corollary 1.6 does not apply, and in general such $X$ does not enjoy the Oka property even if $C$ is non-hyperbolic. The following example was explained to me by J. Winkelmann.

Example 6.3. There exists a ramified elliptic fibration $\pi: X \rightarrow \mathbb{P}_{1}$ such that $X$ is not dominable by $\mathbb{C}^{2}$ (and hence does not enjoy the Oka property).

Let $Z$ be a hyperelliptic Riemann surface of genus $g \geq 2$, with involution $\sigma \in$ $\operatorname{Aut}(Z)$. Then $Z$ is hyperbolic and $Z / \sigma=\mathbb{P}_{1}$. Let $E$ be an elliptic curve considered with its group structure, and let $\tau \in E$ be a non-neutral element of order $2(2 \tau=0$ in $E)$. Then $x \rightarrow x+\tau$ is a fixed point free involution on $E$. Take $X=(Z \times E) / \Gamma$ where $\Gamma$ is the cyclic group of automorphisms generated by $\gamma(z, x)=(\sigma(z), x+\tau)$ which acts without fixed points on $Z \times E$. Let $\pi: X \rightarrow \mathbb{P}_{1}$ be the ramified elliptic fibration induced by the projection $p: Z \times E \rightarrow Z$. We have a commutative diagram

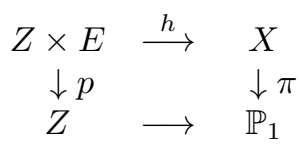

where $h$ is an unramified holomorphic covering. A holomorphic map $f: \mathbb{C}^{n} \rightarrow X$ lifts to a map $g: \mathbb{C}^{n} \rightarrow Z \times E$ such that $f=h \circ g$. Since $Z$ is hyperbolic, the image of $g$ is contained in a fiber of $p$, and hence the map $\pi \circ f: \mathbb{C}^{n} \rightarrow \mathbb{P}_{1}$ is constant. Thus $X$ is not dominable by $\mathbb{C}^{2}$. (In this example $\operatorname{kod} X=1$.)

This concludes our discussion of elliptic fibrations (6.2.4).

Every compact complex surface bimeromorphic to a Kummer K3 surface (in the class $(6.2 .3)$ ) is holomorphically dominable by $\mathbb{C}^{2}$ (proposition 4.5 in [6]). We do not know whether any (or all) Kummer K3 surfaces enjoy the Oka property.

Problem 6.4. To what extent is the Oka property invariant under proper modifications of (projective) algebraic manifolds? In particular, is the Oka property invariant under blowing up of points and/or blowing down of exceptional divisors? 
The answer to the first question is affirmative for manifolds of Class $\mathcal{A}$ discussed in $\S 2$ above, for the simple reason that this class is closed under blowing up points.

6.3. Complements of complex subvarieties. Recall that the complement $\mathbb{P}_{p} \backslash A$ of a thin algebraic subvariety is is algebraically subelliptic (corollary 2.4) and hence enjoys the Oka property. Call a complex subvariety $A \subset \mathbb{C}^{p}$ tame if its closure in $\mathbb{P}_{p}$ does not contain the hyperplane at infinity. The complement $\mathbb{C}^{p} \backslash A$ of a thin tame subvariety is elliptic by lemma 7.1 in [29].

Turning to more general surfaces, we have the following result proved in [26]. Note that the manifolds $X$ in proposition 6.5 include all complex tori, in particular all Abelian varieties.

Proposition 6.5. (Corollary 1.5 (ii) in [26]) Let $X=\mathbb{C}^{p} / \Gamma$ where $\Gamma$ is a lattice in $\mathbb{C}^{p}$ for some $p \geq 2$. For any finite set $x_{1}, \ldots, x_{m} \in X$ the manifold $X_{0}=$ $X \backslash\left\{x_{1}, \ldots, x_{m}\right\}$ enjoys the Oka property.

Proof. Since [26] has not been printed yet, we reproduce here the short proof. Let $\pi: \mathbb{C}^{p} \rightarrow X$ denote the quotient projection. Choose points $q_{j} \in \mathbb{C}^{p}$ with $\pi\left(q_{j}\right)=x_{j}$ for $j=1, \ldots, m$. The discrete set $\Gamma_{0}=\cup_{j=1}^{m}\left(\Gamma+q_{j}\right)$ is tame in $\mathbb{C}^{p}$ (proposition 4.1 in [6], [5]) and hence $\widetilde{X}=\mathbb{C}^{p} \backslash \Gamma_{0}$ is elliptic. Since $\pi: \widetilde{X} \rightarrow X_{0}$ is an unramified covering, the Oka property descends from $\widetilde{X}$ to $X_{0}$ by corollary 1.6

The complement of a complex hypersurface of sufficiently large degree in $\mathbb{P}_{n}$ tends to be hyperbolic (see e.g. 10, [1], 12, [15, 65], 66, 667). On the other hand, the complement of a smooth cubic curve in $\mathbb{P}_{2}$ is dominable by $\mathbb{C}^{2}$ according to proposition 5.1 in [6].

Problem 6.6. (Complements of cubics) Does the complement of a smooth cubic curve $C$ in $\mathbb{P}_{2}$ enjoy the Oka property?

It can be shown that an affirmative answer to the following problem for finitely sheeted maps $\pi$ would imply an affirmative answer to problem 6.6

Problem 6.7. (Descent of the Oka property) Let $\pi: X \rightarrow X_{0}$ be a proper holomorphic map with the ramification locus br $\pi$. Assume that $\pi$ is a subelliptic submersion over $X_{0} \backslash \pi(\mathrm{br} \pi)$ (definition 2.1). Does the Oka property of $X$ imply the Oka property of $X_{0}$ ? What is the answer for finitely sheeted $\pi$ ?

Here is an even more basic question.

Problem 6.8. (Complements of points) Let $X$ be a complex manifold of dimension $\geq 2$ satisfying the Oka property. Does $X \backslash\left\{x_{0}\right\}$ enjoy the Oka property for every point $x_{0} \in X$ ?

The answer is affirmative for algebraic manifolds of Class $\mathcal{A}$ (corollary 2.4), and the author is not aware of any counterexample.

Acknowledgement. I express my sincere thanks to G. Buzzard, F. Kutzschebauch, F. Lárusson and D. Varolin for helpful discussions. I especially thank J. Winkelmann who explained me example 6.3 and the fact, mentioned in $\S 2$, that an algebraic Lie group without a homomorphism to $\mathbb{C}_{*}$ is algebraically elliptic. I also thank D. Trotman for pointing out the references [44] and [55], and the referee for thoughtful remarks which helped me to improve the presentation. 


\section{REFERENCES}

1. ABRAhAm, R., Transversality in manifolds of mappings. Bull. Amer. Math. Soc., 69 (1963), 470-474.

2. BARTH, W., HUlEK, K., PETERS, C. A. M., VAN DE VEN, A., Compact Complex Surfaces, 2nd ed. Springer-Verlag, Berlin, 2004.

3. BISHOP, E., Mappings of partially analytic spaces. Amer. J. Math., 83 (1961), 209-242.

4. BRODY, R., Compact manifolds and hyperbolicity. Trans. Amer. Math. Soc., 235 (1978), 213-219.

5. BUZZARD, G., Tame sets, dominating maps, and complex tori. Trans. Amer. Math. Soc., 355 (2002), 2557-2568.

6. BUZZARD, G., LU, S. S. Y., Algebraic surfaces holomorphically dominable by $\mathbb{C}^{2}$. Invent. Math., 139 (2000), 617-659.

7. CARLSON, J., GRIFFITHS, P., A defect relation for equidimensional holomorphic mappings between algebraic varieties. Ann. Math., (2) 95 (1972), 557-584.

8. CARTAN, H., Espaces fibrés analytiques, in Symposium Internat. de topologia algebraica, 97-121, Mexico, 1958. Oeuvres, Vol. 2, Springer-Verlag, New York, 1979.

9. DEMAILLY, J.-P., Cohomology of $q$-convex spaces in top degrees. Math. Z., 204 (1990), 283-295.

10. - Algebraic criteria for Kobayashi hyperbolic projective varieties and jet differentials. Algebraic Geometry - Santa Cruz 1995, pp. 285-360, Proc. Symp. Pure Math., 62, Part 2, Amer. Math. Soc., Providence, RI, 1997.

11. - Variétés projectives hyperboliques et équations différentielles algébriques. Journée en l'Honneur de Henri Cartan, pp. 3-17, SMF Journ. Annu., 1997, Soc. Math. France, 1997.

12. DEMAILLY, J.-P., El GOUL, J., Hyperbolicity of generic surfaces of high degrees in projective 3-space. Amer. J. Math., 122 (2000), 515-546.

13. DOCQUIER, F., GRAUERT, H., Levisches Problem und Rungescher Satz für Teilgebiete Steinscher Mannigfaltigkeiten. Math. Ann., 140 (1960), 94-123.

14. DEMAILLY, J.-P., LEMPERT, L., SHIFFMAN, B., Algebraic approximations of holomorphic maps from Stein domains to projective manifolds. Duke Math. J., 76 (1994), 333-363.

15. DETHLOFF, G., ZAIDENBERG, M., Plane curves with hyperbolic and C-hyperbolic complements. Ann. Scuola Norm. Sup. Pisa Cl. Sci. (4) 23 (1996), 749-778.

16. EISENMAN, D. A., Intrinsic measures on complex manifolds and holomorphic mappings. Memoirs of the Amer. Math. Soc., 96, American Mathematical Society, Providence, Rhode Island, 1970.

17. ELIASHBERG, Y., GROMOV, M., Nonsingular maps of Stein manifolds. Func. Anal. Appl., 5 (1971), 82-83.

18. — Embeddings of Stein manifolds. Ann. Math., 136 (1992), 123-135.

19. FORSTER, O., Plongements des variétés de Stein. Comment. Math. Helv., 45 (1970), 170184.

20. FORSTER, O., RAMSPOTT, K. J., Analytische Modulgarben und Endromisbündel. Invent. Math., 2 (1966), 145-170.

21. FORSTNERIČ, F., Actions of $(\mathbb{R},+)$ and $(\mathbb{C},+)$ on complex manifolds. Math. Z., 223 (1996), 123-153.

22. — The Oka principle for sections of subelliptic submersions. Math. Z., 241 (2002), $527-551$.

23. - Noncritical holomorphic functions on Stein manifolds. Acta Math., 191 (2003), $143-189$.

24. - Holomorphic submersions from Stein manifolds. Ann. Inst. Fourier, 54 (2004), $1913-1942$

25. - The homotopy principle in complex analysis: A survey. Explorations in Complex and Riemannian Geometry: A Volume dedicated to Robert E. Greene, pp. 73-99, Contemporary Mathematics, 332, American Mathematical Society, Providence, 2003.

26. - Runge approximation on convex sets implies Oka's property. Annals of Math., to appear. [arXiv: math.CV/0402278

27. - Extending holomorphic mappings from subvarieties in Stein manifolds. Ann. Inst. Fourier, 55 (2005), no. 3. [arXiv: math.CV/0411048 
28. FORSTNERIČ, F., PREZELJ, J., Oka's principle for holomorphic fiber bundles with sprays. Math. Ann., 317 (2000), 117-154.

29. - Oka's principle for holomorphic submersions with sprays. Math. Ann., 322 (2002), 633-666.

30. — Extending holomorphic sections from complex subvarieties. Math. Z., 236 (2001), 43-68.

31. GORESKY, M., Whitney stratified chains and cochains. Trans. Amer. Math. Soc., 261 (1981), $175-196$.

32. GORESKY, M., MacPHERSON, R., Stratified Morse Theory. Springer-Verlag, Berlin-New York, 1988.

33. GRAUERT, H., Approximationssätze für holomorphe Funktionen mit Werten in komplexen Räumen. Math. Ann., 133 (1957), 139-159.

34. - Holomorphe Funktionen mit Werten in komplexen Lieschen Gruppen. Math. Ann., 133 (1957), 450-472.

35. — Analytische Faserungen über holomorph-vollständigen Räumen. Math. Ann., 135 (1958), 263-273.

36. GRAUERT, H., KERNER, H., Approximation von holomorphen Schnittflächen in Faserbündeln mit homogener Faser. Arch. Math. 14 (1963), 328-333.

37. GRAUERT, H., REMMERT, R., Theory of Stein Spaces. Grundl. Math. Wiss. 227, Springer, New York, 1977.

38. GREEN, M., GRIFFITHS, P., Two applications of algebraic geometry to entire holomorphic mappings. The Chern Symposium 1979 (Proc. Internat. Sympos., Berkeley, CA, 1979, pp. 41-74), Springer-Verlag, New York, 1980.

39. GROMOV, M., Partial Differential Relations. Ergebnisse der Mathematik und ihrer Grenzgebiete (3), 9, Springer-Verlag, Berlin-Heidelberg-New York, 1986.

40. — Oka's principle for holomorphic sections of elliptic bundles. J. Amer. Math. Soc., 2 (1989), 851-897.

41. GUNNING, R. C., ROSSI, H., Analytic functions of several complex variables. Prentice-Hall, Englewood Cliffs, 1965.

42. HEINZNER, P., KUTZSCHEBAUCH, F., An equivariant version of Grauert's Oka principle. Invent. Math., 119 (1995), 317-346.

43. HENKIN, G., LEITERER, J., The Oka-Grauert principle without induction over the basis dimension. Math. Ann., 311 (1998), 71-93.

44. KALIMAN, S., ZAIDENBERG, M., A transversality theorem for holomorphic mappings and stability of Eisenman-Kobayashi measures. Trans. Amer. Math. Soc., 348 (1996), 1-12.

45. KLEIMAN, S. L., Bertini and his two fundamental theorems. Studies in the history of modern mathematics, III. Rend. Circ. Mat. Palermo, (2), 55 (1998), 9-37.

46. KOBAYASHI, S., Hyperbolic Manifolds and Holomorphic Mappings. Marcel Dekker, New York, 1970

47. - Intrinsic distances, measures and geometric function theory. Bull. Amer. Math. Soc., 82 (1976), 357-416.

48. KOBAYASHI, S., OCHIAI, T., Meromorphic mappings onto compact complex spaces of general type. Invent. Math., 31 (1975), 7-16.

49. KODAIRA, K., Pluricanonical systems on algebraic surfaces of general type. J. Math. Soc. Japan, 20 (1968), 170-192.

50. - Holomorphic mappings of polydiscs into compact complex manifolds. J. Diff. Geom., $6(1971 / 72), 33-46$

51. LÁRUSSON, F., Model structures and the Oka principle. J. Pure Appl. Algebra, 192 (2004), 203-223.

52. - Mapping cylinders and the Oka principle. Preprint, 2004.

http://www.math.uwo.ca/ larusson/papers/

53. LEITERER, J., Holomorphic Vector Bundles and the Oka-Grauert Principle. Encyclopedia of Mathematical Sciences, 10, 63-103, Several Complex Variables IV, Springer-Verlag, BerlinHeidelberg-New York, 1989.

54. LEMPERT, L., Algebraic approximations in analytic geometry. Invent. Math., 121 (1995), $335-354$.

55. MUROlO, C., TROTMAN, D. J. A., DU PLESSIS, A. A., Stratified transversality by isotopy. Trans. Amer. Math. Soc., 355 (2003), 4881-4900. 
56. NARASIMHAN, R., Imbedding of holomorphically complete complex spaces. Amer. J. Math., 82 (1960), 917-934.

57. NASH, J., Real algebraic manifolds. Ann. Math., 56 (1952), 405-421.

58. POLETSKY, E. A., SHABAT, B. V., Invariant metrics. Encyclopedia of Mathematical Sciences, 9, 63-111, Several Complex Variables III, Springer-Verlag, Berlin-Heidelberg-New York, 1989.

59. REMMERT, R., Sur les espaces analytiques holomorphiquement séparables et holomorphiquement convexes. C. R. Acad. Sci. Paris, 243 (1956), 118-121.

60. SARD, A., The measure of critical values of differentiable maps. Bull. Amer. Math. Soc., 48 (1942), 883-890.

61. SCHÜRMANN, J, Embeddings of Stein spaces into affine spaces of minimal dimension. Math. Ann., 307 (1997), 381-399.

62. SERRE, J.-P., Faisceaux algébriques cohérents. Ann. Math., 61 (1955), 197-278.

63. - Géométrie algébrique et géométrie analytique. Ann. Inst. Fourier, 6 (1956), 1-42.

64. SIU, J.-T., Every Stein subvariety admits a Stein neighborhood. Invent. Math., 38 (1976), $89-100$.

65. - Recent techniques in hyperbolicity problems. Several Complex Variables (Berkeley, CA, 1995-1996, pp. 429-508), Math. Sci. Res. Inst. Publ., 37, Cambridge Univ. Press, Cambridge, 1999.

66. SIU, Y.-T., YEUNG, S.-K., A generalized Bloch's theorem and the hyperbolicity of the complements of an ample divisor in an abelian variety. Math. Ann., 306 (1996), 743-758; Erratum, Math. Ann., 326 (2003), 205-207.

67. — , Defects for ample divisors of abelian varieties, Schwarz lemma, and hyperbolic surfaces of low degrees. Amer. J. Math., 119 (1997), 1139-1172; Addendum, Amer. J. Math., 125 (2003), 441-448.

68. THOM, R., Les singularités des applications differentiables. Ann. Inst. Fourier, 6 (1955), $43-87$.

69. - Un lemme sur les applications différentiables. Bol. Soc. Mat. Mexicana, (2) 1 (1956), $59-71$.

70. TROTMAN, D., Stability of transversality to a stratification implies Whitney (a)-regularity. Invent. Math., 50 (1979), 273-277.

71. WHITEHEAD, G. W., Elements of Homotopy Theory. Graduate Texts in Math., 61, SpringerVerlag, 1978.

72. WHITNEY, H., Local properties of analytic varieties. Differentiable and Combinatorial Topology, A Symposium in honor of Marston Morse (S. Cairns, ed., 205-244), Princeton University Press, Princeton, N.J., 1965.

73. WINKELMANN, J., The Oka-principle for mappings between Riemann surfaces. L'Enseignement Math., 39 (1993), 143-151.

74. ZALCMAN, L., A heuristic principle in complex function theory. Amer. Math. Monthly, 82 (1975), 813-817.

Institute of Mathematics, Physics and Mechanics, University of Ljubljana, JadranSKa 19, 1000 Ljubluana, Slovenia

E-mail address: franc.forstneric@fmf.uni-lj.si 